TRANSACTIONS OF THE

AMERICAN MATHEMATICAL SOCIETY

Volume 351, Number 1, January 1999, Pages 131-169

S 0002-9947(99)02124-8

\title{
CLASSIFYING 2-GROUPS BY COCLASS
}

\author{
M. F. NEWMAN AND E. A. O'BRIEN
}

\begin{abstract}
Now that the conjectures of Leedham-Green and Newman have been proved, we probe deeper into the classification of $p$-groups using coclass We determine the pro-2-groups of coclass at most 3 and use these to classify the 2-groups of coclass at most 3 into families. Using extensive computational evidence, we make some detailed conjectures about the structure of these families. We also conjecture that the 2-groups of arbitrary fixed coclass exhibit similar behaviour.
\end{abstract}

\section{INTRODUCTION}

The idea of classifying groups of prime-power order by coclass has proved to be very fruitful. Recall that the coclass of a group of order $p^{n}$ and class $c$ is $n-c$.

Leedham-Green \& Newman (1980) made a detailed series of conjectures about groups of prime-power order ( $p$-groups) using coclass as the primary invariant. Leedham-Green (1994) and Shalev (1994) have proved a number of important theorems as the culmination of the program of studying and confirming these conjectures.

A feature of this program is to study pro- $p$-groups of finite coclass. A pro-p-group of coclass $r$ is an inverse limit of (an infinite chain of) finite $p$-groups of coclass $r$. Leedham-Green, McKay \& Plesken (1986a, 1986b) proved that, given a prime $p$ and a positive integer $r$, there are finitely many soluble pro- $p$-groups of coclass $r$ (Theorem E); a strengthened version of their result was proved by McKay (1994). Shalev \& Zel'manov (1992) proved that pro- $p$-groups of finite coclass are soluble (Theorem $\mathrm{C}$ ). Hence there are finitely many pro- $p$-groups of coclass $r$.

Recall, from Leedham-Green \& Newman (1980), that we can define a directed graph $\mathcal{G}_{p}$ on $p$-groups. Its vertices are all $p$-groups for a fixed prime $p$, one for each isomorphism type, and its edges are the pairs $(P, Q)$, with $P$ isomorphic to the quotient $Q / \gamma_{c}(Q)$ where $\gamma_{c}(Q)$ is the last non-trivial term of the lower central series of $Q$. If $(P, Q)$ is an edge in $\mathcal{G}_{p}$, then $Q$ is an immediate descendant of $P$. We say that $R$ is a descendant of $P$ if there is a (possibly empty) path from $P$ to $R$ in $\mathcal{G}_{p}$. A group is capable if it has immediate descendants; otherwise it is terminal. The descendant tree of $P$ is the subgraph of its descendants. A group of class $c$ is infinitely capable if it has descendants of all classes greater than $c$.

Received by the editors November 1, 1996.

1991 Mathematics Subject Classification. Primary 20D15; Secondary 20-04.

Key words and phrases. p-groups, pro-p-groups, coclass.

O'Brien was supported by the Alexander von Humboldt Foundation, Bonn, via a Research Fellowship held at the Lehrstuhl D für Mathematik, RWTH Aachen, and by a Visiting Fellowship at the School of Mathematical Sciences, Australian National University. We thank Rodney James, C.R. Leedham-Green, and W. Plesken for helpful discussions.

(C)1999 American Mathematical Society 
A finite $p$-group is coclass settled if all its descendants have the same coclass. Shalev (1994, Corollary 4.4) has shown that the 2-groups of coclass $r$ are coclass settled by class $2^{r+3}$ and that, for $p$ odd, the $p$-groups of coclass $r$ are coclass settled by class $2 p^{r}$.

We can associate with each pro-p-group $G$ of coclass $r$ a family of finite $p$-groups of coclass $r$ in the following way. All the finite quotients of $G$ have coclass at most $r$. All but finitely many of these are coclass settled and have coclass $r$. Since there are only finitely many pro- $p$-groups of coclass $r$, all but finitely many of the coclass settled finite quotients are quotients of only one pro- $p$-group. These finite quotients form an infinite chain. The family $\mathcal{F}_{G}$ associated with $G$ is the tree of descendants of the smallest group $R_{G}$ in the chain. We call $R_{G}$ the root of the family. An infinitely capable descendant of a root is mainline. It follows from Leedham-Green (1994, Proposition 2.2) that all but finitely many $p$-groups of coclass $r$ belong to some family.

Descriptions are known for a small number of families. The unique family of 2 -groups of coclass 1 is well-known: for $n \geq 4$, the number of isomorphism types of order $2^{n}$ is 3 . In fact, there is exactly one family of $p$-groups of coclass 1 for every prime $p$. Blackburn (1958) described this family for the prime 3: for $n \geq 3$, the number of 3 -groups of order $3^{2 n-1}$ is 6 and of order $3^{2 n}$ is 7 . The family of 5-groups of coclass 1 is conjecturally described by Newman (1990). It is clear from Leedham-Green \& McKay (1984) that the descriptions are more complex as the prime increases. The 2-groups of coclass 2 have been studied by James (1975, 1983). His results - with corrections presented here - can be summarised as follows: for $n \geq 4$, the number of 2 -groups of coclass 2 and order $2^{2 n-1}$ is 29 and of order $2^{2 n}$ is 38 . There is also unpublished work of Leedham-Green and Newman on the structure of the families of 3 -groups of coclass 2 .

The results of Leedham-Green (1994) and Shalev (1994) suggest that the families of 2-groups of finite coclass will be easier to describe than the families for odd primes. One formulation of Theorem A is the following: there exists a function $f(p, r)$ such that every $p$-group $P$ of coclass $r$ has a normal subgroup $A$ of order bounded by $f(p, r)$ and $P / A$ is constructible. The constructible 2-groups are mainline (from space groups).

Leedham-Green (1994, Definition 1.3) produced an internal criterion for a $p$ group to be coclass settled. He introduced the notion of a finite $p$-group being settled and proved that every settled group is coclass settled and that all descendants of a settled group are settled.

For practical computational reasons, we use a variation of the lower central series known as the lower exponent-p central series. This is the descending sequence of subgroups

$$
G=\mathcal{P}_{0}(G) \geq \ldots \geq \mathcal{P}_{i-1}(G) \geq \mathcal{P}_{i}(G) \geq \ldots
$$

where $\mathcal{P}_{i}(G)=\left[\mathcal{P}_{i-1}(G), G\right] \mathcal{P}_{i-1}(G)^{p}$ for $i \geq 1$. By direct analogy, we define the lower exponent- $p$ class and lower exponent- $p$ coclass of $G$. A group of lower exponent- $p$ class $c$ has nilpotency class at most $c$. If both the nilpotency coclass and lower exponent- $p$ coclass of a $p$-group $G$ are finite and $G$ has sufficiently large order, then the two coclasses are the same; see Theorem 6.1. We use the lower exponent- $p$ coclass of a $p$-group as a classification invariant and in the remainder of the paper refer to lower exponent- $p$ coclass simply as coclass. 
We now present a modification of settled which applies for lower exponent- $p$ coclass.

Definition 1.1. A $p$-group $P$ of class $c$ is settled with respect to a normal subgroup $P_{1}$ of order $p^{t}$ if

(a) all the $P$-invariant subgroups $P_{2}, P_{3}, \ldots$ of $P_{1}$ are totally ordered by inclusion where

$$
P_{1}>P_{2}>\cdots>P_{t}>P_{t+1}=E=P_{t+2}=\ldots ;
$$

(b) $\left\langle x^{p}: x \in P_{i}\right\rangle=P_{i+s}$ for some fixed positive $s$ and for all $i \geq 1$;

(c) $P_{t}=\mathcal{P}_{c-1}(P)$

(d) $(p-1) t>p s$.

Our next result generalises Theorem 1.6 of Leedham-Green (1994); a proof is given in Section 6 .

Theorem 1.2. All the descendants of a settled group are settled and have the same coclass.

There are examples of coclass settled groups which are not settled.

Armed with Theorem 1.2, we can now adapt the various notions introduced earlier in the nilpotency context to the exponent- $p$ coclass context. In particular, we define the graph $\mathcal{G}_{p}$ and its related concepts in terms of the lower exponent- $p$ central series. There are finitely many families of coclass $r$ and all but finitely many $p$-groups of coclass $r$ belong to some family. We call the exceptions sporadic.

We illustrate these concepts by reference to the 2-groups of coclass 1 . There are two families of these groups. The first has root $C_{2} \times C_{4}$ and two groups of order $2^{n}$ for every $n \geq 4$. The second, already mentioned above, has root $D_{8}$, the dihedral group of order 8; we consider this family in Section 5.1. The only sporadic 2-generator 2-groups of coclass 1 are $C_{2} \times C_{2}$ and $Q_{8}$, the quaternion group of order 8. The first of these is not coclass settled, the other has finitely many descendants. There are sporadic groups which are both coclass settled and have infinitely many descendants; they have more than one root as a descendant. The quotient of order $2^{5}$ of the pro-2-group considered in Section 5.2 is such a group.

We report here on an extensive investigation of the 2-groups of coclass 3 and prove that there are 70 such families; in Table 2 we identify 54 families which have nilpotency coclass 3 . (Unpublished work of James agrees with this.) We also include results for the 2 -groups of coclass 2 . The computations which underpinned our investigation were first carried out in 1986; our interpretation of them is influenced by the recent results of Leedham-Green (1994) and Shalev (1994).

For each of the pro-2-groups, we have established that their 2-quotients of order $2^{15}$ are settled. Theorem 1.2 now implies that all groups in the descendant tree of each mainline group of order $2^{15}$ are coclass settled. Using our implementation of the $p$-group generation algorithm, we have determined the tree of 2-groups of coclass 3 as far as all groups of order $2^{23}$ and in places well beyond that. Combining this information, we obtain that all 2-groups of coclass 3 and order at least $2^{10}$ are coclass settled; there are groups of order $2^{9}$ which are not coclass settled.

The descendant tree $\mathcal{T}_{P}$ of a $p$-group $P$ is periodic if $P$ has a proper descendant $Q$ such that $\mathcal{T}_{Q}$ is isomorphic to $\mathcal{T}_{P}$. The period of a periodic $\mathcal{T}_{P}$ is the least value of $\log _{p}(|Q| /|P|)$ and the descendant pattern of $\mathcal{T}_{P}$ is $\mathcal{T}_{P}-\mathcal{T}_{Q}$. 
Our study suggests that each of the 70 families of 2 -groups of coclass 3 has a periodic tree structure. In 59 cases we conjecture that the descendant tree of the root of the family is already periodic. For the remaining families, the conjectured periodic behaviour is observable in the descendant tree of a mainline group of order at most $2^{3}$ greater than that of the root. For each family we define its periodic root as the smallest infinitely capable descendant of the root whose descendant tree is periodic. The roots of the families have order at most $2^{11}$ and the conjectured periodic root has order at most $2^{14}$. We conjecture that the period for each family divides 4 . There are 1782 sporadic 2 -groups of coclass 3 and these have order at most $2^{14}$. Similar periodic behaviour is already known for the 2-groups of coclass at most 2 .

The (conjectured) descendant patterns for the families are presented in Appendix B. In Section 5 we show how to verify these conjectures in two cases. It should be possible to use similar arguments to deal with the remaining cases, although the conjectured patterns make this a daunting prospect. Our proofs are effectively applications of the $p$-group generation algorithm to groups of order $2^{n}$, for arbitrary $n$. For details of the algorithm and terminology, we refer the reader to O'Brien (1990). In an attempt to reduce some of the tedium inherent in such calculations, we introduce the concept of a universal group for a family; this group has all the groups in the family as quotients and is minimal with respect to this property. Our conjectured descendant patterns imply the existence of a universal group for each family.

We now formulate some more general conjectures arising from these observations.

Conjecture P. Each family of 2-groups of coclass $r$ has a periodic root $R$ and $R$ has a descendant $Q$ of order dividing $2^{r-1}|R|$ such that the descendant tree of $Q$ is isomorphic to the descendant tree of $R$.

A corollary to Conjecture $\mathrm{P}$ is that, for sufficiently large $n$, the number of isomorphism types of 2-groups of coclass at most $r$ with order $2^{n}$ is the same as the number with order $2^{n+2^{r-1}}$ (see Problem 27 of Shalev, 1995).

Conjecture Q. All 2-groups of coclass $r$ and order $2^{2^{r+1}}$ are (coclass) settled.

Conjecture $\mathbf{R}$. The periodic roots of the families of 2-groups of coclass $r$ have order at most $2^{2^{r+1}}$.

Conjecture S. All sporadic 2-groups of coclass $r$ have order at most $2^{2^{r+2}}$.

We do not expect that the conjectures hold in this form for odd primes. A more complex notion of periodicity is conjectured for the 5 -groups of coclass 1 by Newman (1990); detailed conjectures for odd primes are formulated by Schneider (1997).

Leedham-Green (1994) proved that all but a finite number of 2-groups $P$ of coclass $r$ have a normal subgroup $A$ of order at most $2^{2^{r-1}\left(2^{r-1}+r+3\right)}$ such that $P / A$ is mainline. Shalev (1995, Problem 26) asked for explicit bounds on the order of $A$. It is easy to construct examples of 2-groups of coclass $r$ where $A$ has order $2^{2^{r-1}}$. For the 2-groups of coclass 1 and 2, the precise bounds are 2 and 4 respectively. The conjectures presented in Appendix B imply that an upper bound for the 2-groups of coclass 3 is 64; Family \#19 has groups where $A$ has order 64 . 
In order to provide access to our classification of the 2-groups of coclass at most 3 , we have prepared an electronic file containing the presentations for the pro-2groups. These presentations may be used in GAP (Schönert et al., 1997) or MAGMA (Bosma \& Cannon, 1997) to obtain power-commutator presentations for the root of each family or for larger (mainline) 2-quotients. Our implementation of the $p$ group generation algorithm can be used in either system to generate parts of the associated trees.

The structure of the paper is as follows. In Section 2 we show that there are 82 pro-2-groups of coclass at most 3 and in Appendix A list explicit pro-2 presentations for these. In Section 3 we summarise results for the 2-groups of coclass 2. In Section 4 we conjecture that there is a periodic tree description for each coclass 3 family. The (conjectured) descendant patterns are presented in Appendix B. In Section 5 we show how to verify these patterns in two cases. Finally we provide a brief description and references for the computational tools used in our investigation.

\section{The PRO-2-GRoups of COClass at MOST 3}

We know there are finitely many pro- $p$-groups of coclass $r$ and they are soluble. On the basis of this we can, in theory, determine (write down pro- $p$ presentations for) all the pro- $p$-groups of coclass $r$. We will show how to do this for pro-2-groups of coclass at most 3 and hence prove the following result.

Theorem 2.1. There are 82 pro-2-groups of coclass at most 3 .

The statement and proof of the following lemma are modelled on Leedham-Green \& Newman (1980, (6) and (9)).

Lemma 2.2. A finitely generated pro-p-group of coclass $r$ is either a central extension of a cyclic subgroup of order $p$ by a pro-p-group of coclass $r-1$ or a uniserial p-adic space group.

Proof. Let $G$ be a pro-p-group of coclass $r$ and let

$$
G=\mathcal{P}_{0}(G) \geq \ldots \geq \mathcal{P}_{i-1}(G) \geq \mathcal{P}_{i}(G) \geq \ldots
$$

be its lower exponent- $p$ central series. Let $u$ be the least positive integer such that $\left|G / \mathcal{P}_{u}(G)\right|=p^{u+r}$ and let $K$ be a non-trivial normal subgroup of $G$ which lies in $\mathcal{P}_{u}(G)$. Then $K \leq \mathcal{P}_{v}(G)$ and $K \not \leq \mathcal{P}_{v+1}(G)$ for some $v \geq u$. Hence $K \mathcal{P}_{j}(G)=\mathcal{P}_{v}(G)$ for all $j \geq v$. It follows that a finite normal subgroup of $G$ avoids $\mathcal{P}_{u}(G)$, has $p$-power order and contains a normal subgroup $L$ of order $p$. Moreover $G / L$ is a pro-p-group of coclass $r-1$. If $G$ has no non-trivial normal subgroup of finite order, then an argument of Leedham-Green \& Newman $(1980,(9))$, with minor changes, applies to show that $G$ is a uniserial $p$-adic space group.

Using Lemma 2.2, we can now determine the pro-2-groups of coclass at most 3 up to isomorphism. Such a group is either a 2-adic space group of coclass at most 3 or a central extension of a cyclic group of order 2 by a pro-2-group of coclass at most 2 .

McKay (1994) shows that the coclass of a uniserial $p$-adic space group of dimension $p^{(r-1)}(p-1)$ is at least $r$. Hence we only need to consider uniserial 2-adic space groups of ranks 1, 2 and 4 .

Brown et al. (1978) described the space groups of dimensions 2, 3 and 4. Finken (1979) determined the 2-uniserial space groups among these. Another description of the space groups of rank 2 can be found in Lyndon (1985, Chapter 4). 
The group of 2-adic integers $\mathbb{Z}_{2}$ is the unique uniserial 2-adic group of coclass 0 .

The direct product of a cyclic group of order 2 with $\mathbb{Z}_{2}$ is the only pro-2-group of coclass 1 which arises as a central extension of a cyclic group of order 2 . The only uniserial 2-adic space group of coclass 1 is the pro-2 completion of the infinite dihedral group and has pro-2 presentation

$$
\left\{t, a: a^{2}=1, a t a^{-1}=t^{-1}\right\} .
$$

Finken (1979) determined the two 2-uniserial space groups of coclass 2 and rank 2. The other pro-2-groups of coclass 2 are extensions of a cyclic group of order 2 by a pro-2-group of coclass 1 . In total, there are 9 pro-2-groups of coclass 2 .

It follows from Leedham-Green \& Plesken (1986, Theorem IV.4 and IV.5(iv)) that the uniserial 2-adic space groups of rank 4 are completions of 2-uniserial space groups, or have point group $Q_{16}$. Finken (1979) determined the 202 -uniserial space groups of rank 4 and coclass 3 and the one 2-uniserial space group of rank 2 and coclass 3. It can be deduced from McKay (1994) that there is exactly one uniserial 2 -adic space group of rank 4 and coclass 3 whose point group is $Q_{16}$; this group is non-split and can be obtained as a subgroup of index 2 in the corresponding split extension which has coclass 4. A 4-dimensional matrix representation for $Q_{16}$ over the 2-adic integers was provided by Leedham-Green, McKay \& Plesken (1986b). Using the matrices, we can write down a pro-2 presentation for the split group. Successive 2-adic approximations to $\sqrt{-39}$ can now be used to obtain presentations for arbitrarily large mainline 2-quotients of the coclass 3 space group.

For each of the 9 pro-2-groups of coclass 2, it is easy to write down pro-2 presentations for the relevant extensions. As one example, we consider the case of central extensions $G$ of a cyclic group $L$ of order 2 by the group having pro- 2 presentation

$$
\left\{a, t, u \quad: \quad[u, t]=1,[u, a]=1, u^{2}=1, a^{2}=1, a t a^{-1}=t^{-1}\right\} .
$$

The largest finite normal subgroup $M$ of $G$ is either $C_{2} \times C_{2}$ or $C_{4}$. The centraliser $C$ of $M$ in $G$ has index 1 or 2. In the case when $M$ is $C_{2} \times C_{2}$ and $C$ has index 2 and $C / L$ is the translation subgroup of $G / L$, it suffices to consider the pro-2 presentations

$$
\begin{aligned}
\{a, t, u, v: & u^{2}=v^{2}=[u, v]=1,[u, t]=[v, t]=1, \\
& {\left.[v, a]=1,[u, a]=v, a t a^{-1}=t^{-1} u^{\beta} v^{\gamma}, a^{2}=v^{\alpha}\right\} }
\end{aligned}
$$

where each of $\alpha, \beta$ and $\gamma$ is 0 or 1 . Considering $a^{2} t a^{-2}$ shows that $\beta=0$. Replacing $t$ by $t u^{\gamma}$ and $a$ by $a u^{\alpha}$ shows that we can take $\alpha=\gamma=0$. Hence one presentation suffices in this case.

In this way, it is straightforward to reduce the resulting list to 82 presentations. The pairwise non-isomorphism of these 82 groups has been checked by showing the class 11 2-quotients are all different.

TABLE 1. Pro-2-groups by rank and coclass

\begin{tabular}{|c|c|c|c|c|c|}
\hline Coclass & Rank 1- & Rank 1 & Rank 2 & Rank 4 & Total \\
\hline 0 & 1 & & & & 1 \\
\hline 1 & 1 & 1 & & & 2 \\
\hline 2 & 4 & 3 & 2 & & 9 \\
\hline 3 & 16 & 18 & 15 & 21 & 70 \\
\hline
\end{tabular}


Table 1 provides a summary of the numbers of pro-2-groups by coclass and rank. Those which are extensions by $\mathbb{Z}_{2}$ are nilpotent; the others have nilpotency coclass as shown (see Theorem 6.1). To distinguish between these, we say that the pro-2groups obtained as extensions by $\mathbb{Z}_{2}$ have rank $1^{-}$.

In Table 2 we summarise some properties of the 82 pro-2-groups. For each pro2 -group $G$, we record its coclass and rank; $d$ is its minimal number of generators; $\left|R_{G}\right|$ is the order of the root for its associated family. We partially identify $G$ by naming its associated uniserial 2-adic space group and the isomorphism type of the largest finite normal subgroup $M$ of $G$. Note that $G$ is just infinite if and only if $M$, its hyper-centre, is trivial. The uniserial 2-adic space group of coclass 1 and rank 1 is labelled $\mathbb{D}$; the uniserial 2-adic space group of coclass 3 with point group $Q_{16}$ is labelled $\mathbb{Q}$; the others are labelled following the notation of Brown et al. (1978).

Our numbering of the pro-2-groups has no special significance and reflects the sequence in which portions of the descendant trees were generated using our implementation of the $p$-group generation algorithm.

\section{The 2-GRoups of COClASS 2}

The 2-groups of nilpotency coclass 2 were studied by James $(1975,1983)$. We have found an additional problem with one family of his revised list and present a new determination of this family in Section 5.2.

We now summarise our results for the 2 -groups of coclass 2 . The associated trees for the 9 pro-2-groups of coclass 2 have roots of order dividing $2^{6}$. The descendant trees of the roots are periodic and have periods dividing 2 . The class 42 -quotients are coclass settled and there are 23 sporadic groups. The number of isomorphism types for the 2-groups of coclass 2 and order at least $2^{n}$ for $n \geq 7$ is given in Table 3 .

\section{The 2-GRoups of COClASS 3}

We now state precise conjectures for the 2-groups of coclass 3 and in Appendix B provide evidence for these.

Conjecture 4.1. Each of the 70 families of 2-groups of coclass 3 has a periodic root of order at most $2^{14}$ and each period divides 4 .

Conjecture 4.2. For $n \geq 17$, the number of isomorphism types of 2-groups of coclass 3 and order $2^{n+4}$ is the same as the number of order $2^{n}$.

Conjecture 4.3. The number of isomorphism types for the 2-groups of coclass 3 and order at least $2^{n}$ for $n \geq 17$ is given in Table 4 .

Since we have constructed the tree of all 2-groups of coclass 3 up to $2^{23}$, Conjectures 4.2 and 4.3 are theorems for $n \leq 19$. We also have the following result.

Theorem 4.4. There are 1782 sporadic 2-groups of coclass 3 and these have order at most $2^{14}$.

\section{Three Families}

In Appendix B we present conjectured descendant patterns for each of the 70 families of 2-groups of coclass 3; we also list the descendant patterns for the 12 families of 2-groups of coclass at most 2 . We present three examples here to illustrate how these tables are interpreted and for two of these verify their correctness. 
TABLE 2. Summary information for the pro-2-groups

\begin{tabular}{|c|c|c|c|c|c|c|}
\hline Family & Coclass & Rank & $d$ & $\log _{2}\left|R_{G}\right|$ & 2-adic space group & $M$ \\
\hline 0 & 0 & $1^{-}$ & 1 & 1 & $\mathbb{Z}_{2}$ & $C_{1}$ \\
\hline 1 & 1 & $1^{-}$ & 2 & 3 & $\mathbb{Z}_{2}$ & $C_{2}$ \\
\hline 2 & 1 & 1 & 2 & 3 & $\mathbb{D}$ & $C_{1}$ \\
\hline 3 & 2 & $1^{-}$ & 2 & 5 & $\mathbb{Z}_{2}$ & $C_{4}$ \\
\hline 4 & 3 & $1^{-}$ & 2 & 7 & $\mathbb{Z}_{2}$ & $C_{8}$ \\
\hline 5 & 3 & $1^{-}$ & 2 & 7 & $\mathbb{Z}_{2}$ & $C_{8}$ \\
\hline 6 & 2 & $1^{-}$ & 2 & 5 & $\mathbb{Z}_{2}$ & $C_{2} \times C_{2}$ \\
\hline 7 & 2 & 2 & 2 & 6 & $3 / 2 / 1 / 2$ & $C_{1}$ \\
\hline 8 & 2 & 2 & 2 & 6 & $3 / 1 / 1 / 1$ & $C_{1}$ \\
\hline 9 & 2 & 1 & 2 & 5 & $\mathbb{D}$ & $C_{2}$ \\
\hline 10 & 3 & $1^{-}$ & 2 & 7 & $\mathbb{Z}_{2}$ & $C_{2} \times C_{2} \times C_{2}$ \\
\hline 11 & 3 & 4 & 2 & 11 & $32 / 13 / 3 / 2$ & $C_{1}$ \\
\hline 12 & 3 & 4 & 2 & 11 & $32 / 7 / 2 / 3$ & $C_{1}$ \\
\hline 13 & 3 & 4 & 2 & 10 & $32 / 2 / 2 / 2$ & $C_{1}$ \\
\hline 14 & 3 & 4 & 2 & 10 & $26 / 1 / 1 / 1$ & $C_{1}$ \\
\hline 15 & 3 & 4 & 2 & 10 & $32 / 13 / 3 / 4$ & $C_{1}$ \\
\hline 16 & 3 & 4 & 2 & 10 & $32 / 13 / 3 / 5$ & $C_{1}$ \\
\hline 17 & 3 & 4 & 2 & 10 & $32 / 7 / 1 / 3$ & $C_{1}$ \\
\hline 18 & 3 & 2 & 2 & 8 & $3 / 2 / 1 / 2$ & $C_{2}$ \\
\hline 19 & 3 & 4 & 2 & 9 & $32 / 2 / 1 / 2$ & $C_{1}$ \\
\hline 20 & 3 & 4 & 2 & 9 & $32 / 7 / 2 / 4$ & $C_{1}$ \\
\hline 21 & 3 & 2 & 2 & 7 & $3 / 1 / 1 / 1$ & $C_{2}$ \\
\hline 22 & 3 & $1^{-}$ & 2 & 7 & $\mathbb{Z}_{2}$ & $C_{2} \times C_{4}$ \\
\hline 23 & 3 & 2 & 2 & 7 & $3 / 1 / 1 / 1$ & $C_{2}$ \\
\hline 24 & 3 & 2 & 2 & 7 & $3 / 2 / 1 / 2$ & $C_{2}$ \\
\hline 25 & 3 & $1^{-}$ & 2 & 7 & $\mathbb{Z}_{2}$ & $D_{8}$ \\
\hline 26 & 3 & 1 & 2 & 7 & $\mathbb{D}$ & $C_{4}$ \\
\hline 27 & 3 & $1^{-}$ & 2 & 6 & $\mathbb{Z}_{2}$ & $Q_{8}$ \\
\hline 28 & 3 & 4 & 2 & 9 & $32 / 13 / 2 / 5$ & $C_{1}$ \\
\hline 29 & 3 & 4 & 2 & 11 & $32 / 15 / 2 / 3$ & $C_{1}$ \\
\hline 30 & 3 & 4 & 2 & 11 & $32 / 13 / 4 / 4$ & $C_{1}$ \\
\hline 31 & 3 & 4 & 2 & 11 & $32 / 8 / 2 / 4$ & $C_{1}$ \\
\hline 32 & 3 & 4 & 2 & 10 & $32 / 3 / 2 / 2$ & $C_{1}$ \\
\hline 33 & 3 & 4 & 2 & 10 & $26 / 2 / 1 / 2$ & $C_{1}$ \\
\hline 34 & 3 & 4 & 2 & 11 & $32 / 15 / 2 / 2$ & $C_{1}$ \\
\hline 35 & 3 & 4 & 2 & 11 & $32 / 13 / 4 / 3$ & $C_{1}$ \\
\hline 36 & 3 & 2 & 2 & 8 & $3 / 1 / 1 / 1$ & $C_{2}$ \\
\hline 37 & 3 & 2 & 2 & 8 & $3 / 2 / 1 / 2$ & $C_{2}$ \\
\hline 38 & 3 & 2 & 2 & 7 & $3 / 2 / 1 / 2$ & $C_{2}$ \\
\hline 39 & 3 & 1 & 2 & 7 & $\mathbb{D}$ & $C_{2} \times C_{2}$ \\
\hline 40 & 3 & 4 & 2 & 9 & $32 / 13 / 2 / 2$ & $C_{1}$ \\
\hline 41 & 3 & 4 & 2 & 10 & $32 / 8 / 2 / 3$ & $C_{1}$ \\
\hline 42 & 3 & 4 & 2 & 10 & $\mathbb{Q}$ & $C_{1}$ \\
\hline 43 & 3 & 4 & 2 & 9 & $32 / 3 / 1 / 2$ & $C_{1}$ \\
\hline 44 & 3 & 2 & 2 & 8 & $3 / 2 / 1 / 2$ & $C_{2}$ \\
\hline
\end{tabular}


TABle 2. (Continued)

\begin{tabular}{|c|c|c|c|c|c|c|}
\hline Family & Coclass & Rank & $d$ & $\log _{2}\left|R_{G}\right|$ & 2-adic space group & $M$ \\
\hline 45 & 3 & 2 & 2 & 8 & $3 / 1 / 1 / 1$ & $C_{2}$ \\
\hline 46 & 3 & 2 & 2 & 7 & $3 / 2 / 1 / 2$ & $C_{2}$ \\
\hline 47 & 3 & 1 & 2 & 7 & $\mathbb{D}$ & $C_{4}$ \\
\hline 48 & 3 & 1 & 2 & 6 & $\mathbb{D}$ & $C_{4}$ \\
\hline 49 & 2 & $1^{-}$ & 2 & 5 & $\mathbb{Z}_{2}$ & $C_{4}$ \\
\hline 50 & 2 & 1 & 2 & 5 & $\mathbb{D}$ & $C_{2}$ \\
\hline 51 & 3 & $1^{-}$ & 2 & 7 & $\mathbb{Z}_{2}$ & $C_{8}$ \\
\hline 52 & 3 & $1^{-}$ & 2 & 7 & $\mathbb{Z}_{2}$ & $C_{8}$ \\
\hline 53 & 3 & 1 & 2 & 7 & $\mathbb{D}$ & $C_{4}$ \\
\hline 54 & 3 & $1^{-}$ & 2 & 6 & $\mathbb{Z}_{2}$ & $C_{2} \times C_{4}$ \\
\hline 55 & 3 & 1 & 2 & 6 & $\mathbb{D}$ & $C_{2} \times C_{2}$ \\
\hline 56 & 3 & 2 & 2 & 7 & $3 / 2 / 1 / 2$ & $C_{2}$ \\
\hline 57 & 3 & 2 & 2 & 7 & $3 / 1 / 1 / 1$ & $C_{2}$ \\
\hline 58 & 2 & $1^{-}$ & 3 & 4 & $\mathbb{Z}_{2}$ & $C_{2} \times C_{2}$ \\
\hline 59 & 2 & 1 & 3 & 4 & $\mathbb{D}$ & $C_{2}$ \\
\hline 60 & 3 & $1^{-}$ & 3 & 6 & $\mathbb{Z}_{2}$ & $C_{2} \times C_{4}$ \\
\hline 61 & 3 & $1^{-}$ & 3 & 6 & $\mathbb{Z}_{2}$ & $C_{2} \times C_{2} \times C_{2}$ \\
\hline 62 & 3 & 2 & 3 & 7 & $3 / 2 / 1 / 2$ & $C_{2}$ \\
\hline 63 & 3 & 2 & 3 & 7 & $3 / 1 / 1 / 1$ & $C_{2}$ \\
\hline 64 & 3 & 1 & 3 & 6 & $\mathbb{D}$ & $C_{2} \times C_{2}$ \\
\hline 65 & 3 & $1^{-}$ & 3 & 6 & $\mathbb{Z}_{2}$ & $C_{2} \times C_{4}$ \\
\hline 66 & 3 & 1 & 3 & 6 & $\mathbb{D}$ & $C_{2} \times C_{2}$ \\
\hline 67 & 3 & $1^{-}$ & 3 & 5 & $\mathbb{Z}_{2}$ & $C_{2} \times C_{4}$ \\
\hline 68 & 3 & $1^{-}$ & 3 & 6 & $\mathbb{Z}_{2}$ & $D_{8}$ \\
\hline 69 & 3 & 1 & 3 & 6 & $\mathbb{D}$ & $C_{4}$ \\
\hline 70 & 3 & $1^{-}$ & 3 & 5 & $\mathbb{Z}_{2}$ & $Q_{8}$ \\
\hline 71 & 3 & 1 & 3 & 6 & $\mathbb{D}$ & $C_{2} \times C_{2}$ \\
\hline 72 & 3 & 2 & 3 & 6 & $3 / 2 / 1 / 1$ & $C_{1}$ \\
\hline 73 & 3 & 1 & 3 & 6 & $\mathbb{D}$ & $C_{4}$ \\
\hline 74 & 3 & 1 & 3 & 6 & $\mathbb{D}$ & $C_{2} \times C_{2}$ \\
\hline 75 & 3 & 1 & 3 & 5 & $\mathbb{D}$ & $C_{4}$ \\
\hline 76 & 3 & 1 & 3 & 5 & $\mathbb{D}$ & $C_{2} \times C_{2}$ \\
\hline 77 & 3 & 1 & 3 & 5 & $\mathbb{D}$ & $C_{4}$ \\
\hline 78 & 3 & 1 & 3 & 6 & $\mathbb{D}$ & $C_{4}$ \\
\hline 79 & 3 & 1 & 3 & 5 & $\mathbb{D}$ & $C_{4}$ \\
\hline 80 & 3 & $1^{-}$ & 4 & 5 & $\mathbb{Z}_{2}$ & $C_{2} \times C_{2} \times C_{2}$ \\
\hline 81 & 3 & 1 & 4 & 5 & $\mathbb{D}$ & $C_{2} \times C_{2}$ \\
\hline
\end{tabular}

5.1. A coclass 1 family. Here, we consider the family of 2-groups of nilpotency coclass 1. The groups are, of course, well-known: the dihedral, quaternion, and semi-dihedral groups. (Our determination is intended to illustrate the general approach, not to replace existing treatments.) The corresponding limit group is the pro-2 completion $\mathbb{D}$ of the infinite dihedral group and has pro- 2 presentation

$$
\left\{t, a: a^{2}=1, a t a^{-1}=t^{-1}\right\} .
$$


TABLE 3. Number of isomorphism types for $m \geq 4$

\begin{tabular}{|l|c|}
\hline Order & Number \\
\hline $2^{2 m-1}$ & 40 \\
\hline $2^{2 m}$ & 49 \\
\hline
\end{tabular}

TABLE 4. Conjectured number of isomorphism types for $m \geq 4$

\begin{tabular}{|c|c|}
\hline Order & Number \\
\hline $2^{4 m+1}$ & 2504 \\
\hline $2^{4 m+2}$ & 2568 \\
\hline $2^{4 m+3}$ & 8632 \\
\hline $2^{4 m+4}$ & 1532 \\
\hline
\end{tabular}

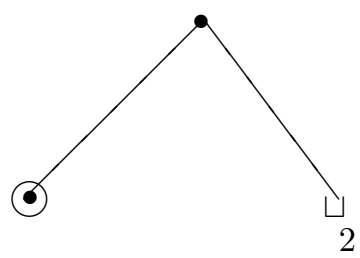

Figure 1. The descendant pattern for Family \#2

TABLE 5. Family \#2 with root order $2^{3}$

\begin{tabular}{|c|c|}
\hline Order & Structure \\
\hline $2^{n+1}$ & $3 / 1$ \\
\hline
\end{tabular}

The root, $R_{G}$, is the dihedral group of order 8 and the structure of $\mathcal{F}_{G}$ can be visualised from Figure 1. This picture can be summarised by Table 5 . In this case, the mainline group of order $2^{n}$, for $n \geq 3$, has three immediate descendants of order $2^{n+1}$, one of which is capable and is the mainline group of order $2^{n+1}$; denoted $3 / 1$.

Our proof for this descendant tree is inductive on $n \geq 3$. We assume that the mainline group $M$ of order $2^{n}$ has power-commutator presentation

$$
\begin{aligned}
\left\{a_{1}, \ldots, a_{n}:\right. & {\left[a_{2}, a_{1}\right]=a_{3},\left[a_{k}, a_{1}\right]=\left[a_{k}, a_{2}\right]=a_{k+1}, } \\
& \left.a_{k}^{2}=a_{k+1} a_{k+2}, 3 \leq k \leq n-1, a_{n-1}^{2}=a_{n}\right\}
\end{aligned}
$$

where all relations whose right-hand sides are trivial are not shown. We verify this claim for $n=3$ by inspecting the power-commutator presentation computed using the $p$-quotient algorithm for the class 22 -quotient of the limit group.

We also assume that a generating set for a supplement to the inner automorphism group of $M$ in the full automorphism group is the following:

$$
\begin{aligned}
\alpha_{1}: \quad a_{1} & \longmapsto a_{2}, \quad \alpha_{k}: \quad \begin{array}{l}
a_{1} \\
a_{2}
\end{array} a_{1} \longmapsto a_{1} \\
a_{2} & \longmapsto a_{2} a_{k+2}
\end{aligned}
$$

where $k=2, \ldots, n-2$. Again, this hypothesis for $n=3$ is readily verified. 
We now determine the immediate descendants of $M$. Its 2-covering group has power-commutator presentation

$$
\begin{aligned}
\left\{a_{1}, \ldots, a_{n}, a_{n+1}, a_{n+2}, a_{n+3}:\right. & {\left[a_{2}, a_{1}\right]=a_{3}, a_{1}^{2}=a_{n+2}, a_{2}^{2}=a_{n+3}, } \\
& {\left[a_{k}, a_{1}\right]=\left[a_{k}, a_{2}\right]=a_{k+1}, } \\
& \left.a_{k}^{2}=a_{k+1} a_{k+2}^{\epsilon_{k+2}}, 3 \leq k \leq n\right\}
\end{aligned}
$$

where

$$
\epsilon_{i}= \begin{cases}1 & \text { if } i \leq n+1 \\ 0 & \text { otherwise }\end{cases}
$$

The consistency of this presentation can be proved using the algorithm described in Sims (1994, Chapter 9).

We now compute the extensions of the automorphisms of $M$ to its 2-covering group. The action of each $\alpha_{k}$ on $a_{j}$, where $3 \leq j \leq n$, is:

$$
a_{j} \longmapsto a_{j} a_{j+k}^{\epsilon_{j+k}}
$$

Their extensions act trivially on the 2-multiplicator of $M$ and play no further role in the computation. The action of $\alpha_{1}$ on $a_{j}$, where $3 \leq j \leq n$, is:

$$
a_{j} \longmapsto a_{j} a_{j+1} \text {. }
$$

Hence the matrix representing the action of $\alpha_{1}$ on the 2-multiplicator of $M$ is:

$$
\left(\begin{array}{lll}
1 & 0 & 0 \\
0 & 0 & 1 \\
0 & 1 & 0
\end{array}\right) \text {. }
$$

The four allowable subgroups have generating sets

$$
\left\{a_{n+1}^{\delta} a_{n+2}, a_{n+1}^{\gamma} a_{n+3}\right\}
$$

where each of $\delta$ and $\gamma$ is 0 or 1 . We label these subgroups from 1 to 4 as usual. The extension of $\alpha_{1}$ permutes these subgroups as $(2,3)$. The resulting 3 orbits, which correspond to distinct isomorphism types, have representatives 1,2 and 4 . We can show that the first of these determines a capable group which has powercommutator presentation and automorphism description like that presented above; hence this group is the mainline group of order $2^{n+1}$. We can also show that the other two descendants are terminal. Hence $M$ has $3 / 1$ descendants.

5.2. A coclass 2 family. Here we present a new determination of the family which was incorrectly determined by James (1983). The limit group is $\mathbb{Z}_{2} \times \mathbb{Z}_{2}$ split by $C_{4}$ acting uniserially and has pro- 2 presentation

$$
\left\{t, a: a^{4}=1,\left(t a^{2}\right)^{2}=1,\left[t, t^{a}\right]=1\right\} .
$$

TABLE 6 . Family \#8 with root order $2^{6}$

\begin{tabular}{|r|r|r|r|}
\hline Order & \multicolumn{3}{|c|}{ Structure } \\
\hline $2^{2 m+1}$ & $6 / 2$ & & \\
\hline $2^{2 m+2}$ & $6 / 2$ & 4 & $\bullet$ \\
\hline $2^{2 m+3}$ & 3 & & \\
\hline
\end{tabular}

The root, $R_{G}$, has order 64 and the structure of $\mathcal{F}_{G}$ can be visualised from Figure 2. This picture can be summarised by Table 6 . In this case, the mainline 


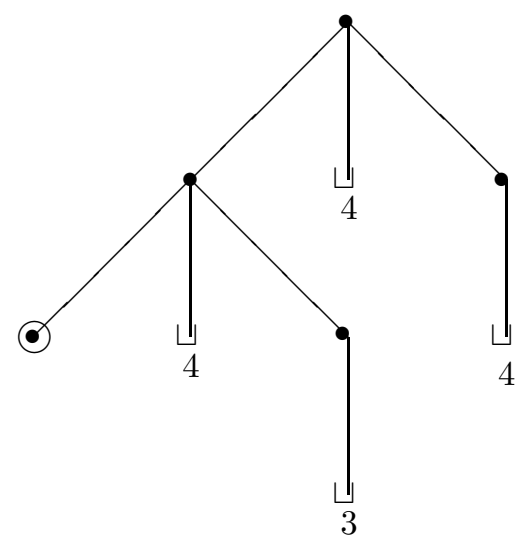

Figure 2. The descendant pattern for Family \#8

group of order $2^{n}$, for $n$ even and at least 6 , has six immediate descendants of order $2^{n+1}$, two of which are capable. One of the two capable groups has six immediate descendants of order $2^{n+2}$, of which two are capable, and the other has four immediate descendants which are terminal. Of the two capable groups of order $2^{n+2}$, one has the same descendant structure as the root; the other has three immediate descendants which are terminal. The $\bullet$ at level $2^{2 m+2}$ in Table 6 indicates that the period is 2 .

Our proof for this descendant tree is inductive on $n$ for $n=2 m$, where $m \geq 3$. We assume that the mainline group $M$ of order $2^{n}$ has power-commutator presentation

$$
\begin{aligned}
\left\{a_{1}, \ldots, a_{n}:\right. & a_{3}=\left[a_{2}, a_{1}\right], a_{4}=a_{1}^{2}, a_{5}=\left[a_{3}, a_{1}\right] \\
& {\left[a_{3}, a_{2}\right]=a_{6} a_{7} a_{8},\left[a_{4}, a_{2}\right]=a_{5} a_{6},\left[a_{4}, a_{3}\right]=a_{6} a_{7}, } \\
& a_{k+1}=\left[a_{k}, a_{1}\right], 5 \leq k \leq n-1, \\
& a_{3}^{2}=a_{6} a_{7}, a_{k}^{2}=a_{k+2} a_{k+3}^{\epsilon_{k+3}}, 5 \leq k \leq n-2, \\
& {\left.\left[a_{k}, a_{2}\right]=\left[a_{k}, a_{4}\right]=a_{k+2} a_{k+3}^{\epsilon_{k+3}} a_{k+4}^{\epsilon_{k+4}}, 5 \leq k \leq n-2\right\} }
\end{aligned}
$$

where $a_{n+1}$ and $a_{n+2}$ are read as the identity, and

$$
\epsilon_{i}= \begin{cases}1 & \text { if } i \leq n, \\ 0 & \text { otherwise }\end{cases}
$$

We verify this claim for $n=6$ by inspecting the power-commutator presentation computed using the $p$-quotient algorithm for the class 42 -quotient of the limit group.

We also assume that a generating set for a supplement to the inner automorphism group of $M$ in the full automorphism group is the following:

$$
\begin{aligned}
& \alpha_{1}: \quad a_{1} \longmapsto a_{1} a_{4}, \quad \alpha_{2}: \quad a_{1} \longmapsto a_{1} a_{2} a_{3}, \quad \alpha_{k}: \quad a_{1} \longmapsto a_{1} \\
& a_{2} \longmapsto a_{2} \quad a_{2} \longmapsto a_{2} a_{5} \quad a_{2} \longmapsto a_{2} a_{k+3}
\end{aligned}
$$

where $k=3, \ldots, n-3$. Again, this hypothesis for $n=6$ can be readily verified. 
We now determine the immediate descendants of $M$. The 2-covering group of $M$ has power-commutator presentation

$$
\begin{aligned}
\left\{a_{1}, \ldots, a_{n}, a_{n+1}, \ldots, a_{n+4}:\right. & a_{3}=\left[a_{2}, a_{1}\right], a_{4}=a_{1}^{2}, a_{5}=\left[a_{3}, a_{1}\right],\left[a_{4}, a_{3}\right]=a_{6} a_{7}, \\
& a_{2}^{2}=a_{n+4}, a_{3}^{2}=a_{6} a_{7} a_{n+2}, a_{4}^{2}=a_{n+3}, \\
& {\left[a_{3}, a_{2}\right]=a_{6} a_{7} a_{8} a_{n+2},\left[a_{4}, a_{2}\right]=a_{5} a_{6} a_{n+2}, } \\
& a_{k+1}=\left[a_{k}, a_{1}\right], 5 \leq k \leq n, \\
& a_{k}^{2}=a_{k+2} a_{k+3}^{\epsilon_{+3}}, 5 \leq k \leq n-1, \\
& {\left.\left[a_{k}, a_{2}\right]=\left[a_{k}, a_{4}\right]=a_{k+2} a_{k+3}^{\epsilon_{k+3}} a_{k+4}^{\epsilon_{k+4}}, 5 \leq k \leq n-1\right\} }
\end{aligned}
$$

where

$$
\epsilon_{i}= \begin{cases}1 & \text { if } i \leq n+1 \\ 0 & \text { otherwise }\end{cases}
$$

The consistency of this presentation can be proved using the algorithm described in Sims (1994, Chapter 9).

An alternative approach to proving that the above presentation is consistent is to write down a universal group for this family. Since we make no use here of the defining property of the universal group - namely, that all groups in the family are quotients of it - we do not establish this claim. This group can be used to simplify other details of our proof. It is obtained as the direct product of the limit group with

$$
\left\{t, a: a^{8}=1,\left(t a^{2}\right)^{4}=1,\left[\left(t a^{2}\right)^{2}, a, t\right]=1, t^{4}=1,[t, a, a]=1\right\},
$$

amalgamating the common quotient defined by

$$
\left\{t, a: a^{4}=1,\left(t a^{2}\right)^{2}=1,\left[t, t^{a}\right]=1, t^{4}=1\right\} .
$$

See Huppert (1967, p. 50) for details of such constructions; they are labelled diagonal products by Conway et al. (1985).

We now compute the extensions of the automorphisms of $M$ to its 2-covering group. The extensions of $\alpha_{3}$ to $\alpha_{n-3}$ act trivially on the 2-multiplicator of $M$ and play no further role in the computation. Under the action of $\alpha_{1}$,

$$
\begin{aligned}
& a_{3} \longmapsto a_{3} a_{5} a_{n+2} \\
& a_{4} \longmapsto a_{4} a_{n+3} .
\end{aligned}
$$

The action of $\alpha_{1}$ on the generators $a_{5}, \ldots, a_{n}$ is:

$$
\begin{aligned}
a_{4 k+1} & \longmapsto a_{4 k+1} a_{4 k+3} a_{4 k+4} a_{4 k+5} \\
a_{4 k+2} & \longmapsto a_{4 k+2} a_{4 k+3} a_{4 k+4} \\
a_{4 k+3} & \longmapsto a_{4 k+3} \\
a_{4 k+4} & \longmapsto a_{4 k+4} a_{4 k+5}
\end{aligned}
$$

where $k \geq 1$ and $a_{i}$ occurs in the image only if $i \leq n+1$. Hence the extended automorphism again induces the identity on the 2-multiplicator of $M$. Finally, consider the action of $\alpha_{2}$ :

$$
\begin{aligned}
& a_{3} \longmapsto a_{3} a_{6} a_{7} \\
& a_{4} \longmapsto a_{3} a_{4} a_{5} a_{7} a_{8} a_{9} a_{n+2} a_{n+4} \\
& a_{5} \longmapsto a_{5} a_{6} a_{7} a_{9} a_{10} a_{11} a_{n+2} .
\end{aligned}
$$


Its action on the generators $a_{6}, \ldots, a_{n}$ is:

$$
\begin{aligned}
a_{4 k-2} & \longmapsto a_{4 k-2} a_{4 k+2} \\
a_{4 k-1} & \longmapsto a_{4 k-1} a_{4 k} a_{4 k+3} a_{4 k+4} \\
a_{4 k} & \longmapsto a_{4 k} a_{4 k+2} a_{4 k+3} \\
a_{4 k+1} & \longmapsto a_{4 k+1} a_{4 k+2} a_{4 k+3} a_{4 k+5} a_{4 k+6} a_{4 k+7}
\end{aligned}
$$

where $k \geq 2$ and again $a_{i}$ occurs in the image of a generator only if $i \leq n+1$. The matrix representing the action of $\alpha_{2}$ on the 2-multiplicator of $M$ is:

$$
\left(\begin{array}{llll}
1 & 0 & 0 & 0 \\
0 & 1 & 0 & 0 \\
0 & 1 & 1 & 0 \\
0 & 0 & 0 & 1
\end{array}\right) .
$$

The eight allowable subgroups have generating sets

$$
\left\{a_{n+1}^{\delta} a_{n+2}, a_{n+1}^{\gamma} a_{n+3}, a_{n+1}^{\psi} a_{n+4}\right\}
$$

where each of $\delta, \gamma, \psi$ is 0 or 1 . We label these subgroups from 1 to 8 as usual. The extension of $\alpha_{2}$ permutes these subgroups as $(2,4)(6,8)$. The resulting 6 orbits, which correspond to distinct isomorphism types, have representatives $1,2,3,5,6$ and 7 . It can be shown, using the universal group, that the first and fourth of these determine capable groups. All of the others can be shown to be terminal. Thus the mainline group has $6 / 2$ immediate descendants.

The first of these capable groups is the mainline group of order $2^{n+1}-$ it has power-commutator presentation and automorphism description like that presented above.

We factor the allowable subgroup

$$
\left\langle a_{n+2}, a_{n+3}, a_{n+1} a_{n+4}\right\rangle
$$

from the 2-covering group of $M$ to give the second group, $Q$ say, of order $2^{n+1}$.

The power-commutator presentation for the 2-covering group of $Q$ has generating set $\left\{a_{1}, \ldots, a_{n+1}, a_{n+2}, \ldots, a_{n+5}\right\}$ and its relations coincide with those for the 2covering group of the mainline group of order $2^{n+1}$ except for the following:

$$
\begin{aligned}
{\left[a_{4}, a_{2}\right] } & =a_{5} a_{6} a_{n+2} a_{n+3} \\
a_{2}^{2} & =a_{n+1} a_{n+5} \\
a_{3}^{2} & =a_{6} a_{7} a_{n+2} a_{n+3} .
\end{aligned}
$$

It can also be shown that a generating set for a supplement to the inner automorphism group of $Q$ is the same as that for the mainline group.

We now determine the immediate descendants of $Q$. Again, the automorphisms, $\alpha_{3}, \ldots, \alpha_{n-2}$, act trivially on the 2-multiplicator of $Q$. The extension of $\alpha_{1}$ acts as:

$$
\begin{aligned}
& a_{3} \longmapsto a_{3} a_{5} a_{n+2} a_{n+3} \\
& a_{4} \longmapsto a_{4} a_{n+4} ;
\end{aligned}
$$

its action on the generators $a_{5}, \ldots, a_{n+1}$ is the same as that described for the mainline group. Therefore,

$$
a_{n+1} \longmapsto \begin{cases}a_{n+1} a_{n+2} & \text { if } n+1 \text { even } \\ a_{n+1} & n+1 \text { odd. }\end{cases}
$$


Hence, if $n+1$ is even, the automorphism matrix is

$$
\left(\begin{array}{llll}
1 & 0 & 0 & 0 \\
0 & 1 & 0 & 0 \\
0 & 0 & 1 & 0 \\
1 & 0 & 0 & 1
\end{array}\right)
$$

and it permutes the allowable subgroups as $(1,5)(2,6)(3,7)(4,8)$. If $n+1$ is odd, the extended automorphism induces the identity.

The extension of $\alpha_{2}$ acts as:

$$
\begin{aligned}
& a_{3} \longmapsto a_{3} a_{6} a_{7} \\
& a_{4} \longmapsto a_{3} a_{4} a_{5} a_{7} a_{8} a_{9} a_{n+1} a_{n+2} a_{n+3} a_{n+5} \\
& a_{5} \longmapsto a_{5} a_{6} a_{7} a_{9} a_{10} a_{11} a_{n+3} ;
\end{aligned}
$$

its action on the generators $a_{6}, \ldots, a_{n+1}$ is the same as that described for the mainline group. Therefore,

$$
a_{n+1} \longmapsto \begin{cases}a_{n+1} & \text { if } n+1 \text { even } \\ a_{n+1} a_{n+2}, & n+1 \text { odd. }\end{cases}
$$

Hence, if $n+1$ is even, the automorphism matrix is

$$
\left(\begin{array}{llll}
1 & 0 & 0 & 0 \\
0 & 1 & 0 & 0 \\
1 & 1 & 1 & 0 \\
0 & 0 & 0 & 1
\end{array}\right)
$$

and it permutes the allowable subgroups as $(1,3)(5,7)$. If $n+1$ is odd, the automorphism matrix is

$$
\left(\begin{array}{llll}
1 & 0 & 0 & 0 \\
0 & 1 & 0 & 0 \\
1 & 1 & 1 & 0 \\
1 & 0 & 0 & 1
\end{array}\right)
$$

and it permutes the allowable subgroups as $(1,7)(2,6)(3,5)(4,8)$.

If $n+1$ is even, the allowable subgroups are permuted by $(1,5)(2,6)(3,7)(4,8)$ and $(1,3)(5,7)$; hence there are three orbits with representatives 1,2 and 4 . If $n+1$ is odd, the allowable subgroups are permuted as $(1,7)(2,6)(3,5)(4,8)$; hence there are four orbits with representatives $1,2,3$ and 4 . It can be shown that all resulting groups are terminal. Thus the non-mainline capable group has $4 / 0$ or $3 / 0$ immediate descendants.

5.3. A coclass 3 family. Here is a pro- 2 presentation for the limit group associated with Family \#43.

$$
\begin{aligned}
\left\{t_{1}, t_{2}, t_{3}, t_{4}, a, b \quad: \quad\right. & {\left[t_{1}, t_{2}\right]=\left[t_{1}, t_{3}\right]=\left[t_{1}, t_{4}\right]=\left[t_{2}, t_{3}\right]=\left[t_{2}, t_{4}\right]=\left[t_{3}, t_{4}\right]=1, } \\
& a^{8}=1, b^{2}=t_{4}, a^{-1} b^{-1} a b a^{6}=t_{1}^{-1} t_{2} t_{3}^{-1}, \\
& a t_{1} a^{-1}=t_{4}, a t_{2} a^{-1}=t_{3}^{-1}, a t_{3} a^{-1}=t_{1}, a t_{4} a^{-1}=t_{2}, \\
& \left.b t_{1} b^{-1}=t_{2}^{-1}, b t_{2} b^{-1}=t_{1}^{-1}, b t_{3} b^{-1}=t_{3}^{-1}, b t_{4} b^{-1}=t_{4}\right\} .
\end{aligned}
$$

The root, $R_{G}$, has order $2^{9}$. We conjecture that the periodic root has order $2^{12}$, that the period is 2 , and that the structure of $\mathcal{F}_{G}$ can be summarised by Table 7 . 
TABLE 7 . Family \#43 with periodic root order $2^{12}$

\begin{tabular}{|c|r|r|r|r|r|r|r|r|}
\hline Order & \multicolumn{7}{|c|}{ Structure } \\
\hline $2^{n+1}$ & $6 / 4$ & & & & & & & \\
\hline $2^{n+2}$ & 4 & $4 / 4$ & $4 / 4$ & 4 & $\bullet$ & & & \\
\hline $2^{n+3}$ & $3 / 2$ & 6 & 3 & $6 / 4$ & 3 & $3 / 2$ & 6 & \\
\hline $2^{n+4}$ & 2 & 2 & 2 & 2 & 2 & 2 & 2 & 2 \\
\hline
\end{tabular}

\section{Proofs}

Theorem 6.1. A p-group $G$ with nilpotency coclass $r$ and order at least $p^{8 p^{r}+r}$ has lower exponent-p coclass $r$.

Proof. Let $\gamma_{m}$ denote the $m$-th term of the lower central series of $G$ and let $\mathcal{P}_{m}$ denote the $m$-th term of the lower exponent- $p$ central series of $G$. So, $G=\gamma_{1}=\mathcal{P}_{0}$. Recall that $\mathcal{P}_{m}=\gamma_{m+1} \gamma_{m}^{p} \ldots \gamma_{1}^{p^{m}}$ (Huppert \& Blackburn, 1982, VIII.1.5). From Shalev (1994, Proposition 4.5), there is a normal subgroup $T$ of $G$ with index at most $p^{8 p^{r}+r-1}$ so that all of the non-trivial factors of the series

$$
T>[T, G] \geq \ldots \geq[T, i G] \geq[T,(i+1) G] \geq \ldots
$$

have order $p$. There is an $n \leq p^{8 p^{r}}$ such that $\gamma_{n}, \gamma_{n-1}^{p}, \ldots$ all lie in $T$. We prove that $\mathcal{P}_{n-1}=\gamma_{n}$ by showing that $\gamma_{n-k}^{p^{k}} \leq \gamma_{n}$ for $k$ in $\{1, \ldots, n-1\}$. Clearly $\gamma_{n}^{p} \leq \gamma_{n+1}$. So $\left[\gamma_{n-1}^{p}, G\right] \leq\left[\gamma_{n}, G\right]$ and hence $\gamma_{n-1}^{p} \leq \gamma_{n}$. An easy induction on $k$ gives the rest. It follows that $G$ has lower exponent- $p$ coclass $r$.

Theorem 1.2. All the descendants of a settled group are settled and have the same coclass.

Proof. Let $P$ be a settled $p$-group as in Definition 1.1. It follows from the definition that $s<t<p(t-s)$.

Let $Q$ be an immediate descendant of $P$, let $\alpha$ be the natural projection of $Q$ onto $P$, and let $Q_{i}$ be the complete inverse image of $P_{i}$ for $1 \leq i \leq t+1$ and $Q_{i}=E$ otherwise. We show that $Q$ is settled with respect to $Q_{1}$.

We prove by induction on $u$ in $\{1, \ldots, s\}$ that $Q_{t-u+2}$ has exponent $p$. If $u=1$, this is obvious. For $u>1$, we have $\left[P_{t-u+1}, P\right]=P_{t-u+2}$ since $\left[P_{t-u+1}, P\right] P_{t-u+1}^{p}$ equals $P_{t-u+2}$ by uniseriality, and $P_{t-u+1}^{p}=E$. It follows that $\left[Q_{t-u+1}, Q\right] Q_{t+1}$ equals $Q_{t-u+2}$. For $h \in Q_{t-u+1}$ and $x \in Q$ we get $[h, x]^{p}=\left[h^{p}, x\right]$ by the usual expansion because $p(t-s)>t$, and the result follows.

Second, we prove that $Q_{t-s+1}$ has exponent $p^{2}$. Clearly $Q_{t-s+1}$ has exponent dividing $p^{2}$; we prove that its exponent is not $p$. By definition, $Q_{t+1}=\left[Q_{t}, Q\right] Q_{t}^{p}$. If $Q_{t}^{p} \neq E$, the result follows. Otherwise, $Q_{t+1}=\left[Q_{t}, Q\right]$. There exists $h \in Q_{t-s}$ with $h^{p} \in Q_{t}-Q_{t+1}$. Hence there exists $x \in Q$ with $\left[h^{p}, x\right] \neq 1$, so $[h, x]^{p} \neq 1$ and $[h, x] \in Q_{t-s+1}$.

Finally, we prove that $Q_{t+1}$ has order $p$. The argument above shows that $Q_{t-s+1}^{p}$ has order $p$. This holds for every immediate descendant of $P$. So $Q_{t-s+1}$ does not lie in any proper normal subgroup of $Q_{t+1}$. Therefore $Q_{t+1}$ has order $p$.

\section{Computational tools}

As already indicated, our conjectures rely on extensive computations. Here we summarise the computational tools used and provide appropriate references. 
Central to our investigation was the ANU p-Quotient Program, which offers access to implementations of the following:

- The $p$-quotient algorithm: it computes power-commutator presentations for $p$-quotients of finitely-presented groups. For further details, see Newman \& O'Brien (1996).

- The $p$-group generation algorithm: it generates the descendants of a $p$-group. For further details, see Newman (1977) and O'Brien (1990).

- The standard presentation algorithm: it computes a canonical or standard presentation for a $p$-group, thereby providing a practical solution to the isomorphism problem for $p$-groups. For further details, see O'Brien (1994).

For a detailed description of the program, see Newman \& O'Brien (1996). Our implementations of these algorithms are also available in GAP and MAGMA. The calculations reported here can be performed using completely routine resources.

\section{Appendix A. Presentations for the PRO-2-Groups}

We list pro- 2 presentations for the 82 pro-2-groups of coclass at most 3 . Our presentations for the uniserial 2-adic space groups of rank dividing 4 are taken from Finken (1979). Much of the work in writing down the presentations for the remaining pro-2-groups was done by Michael Burns while holding a Vacation Scholarship at the Australian National University.
$0 .\{t$
| $\}$
1. $\{t, u$
| $u^{2}=1$,
$\left.t^{-1} u t=u\right\}$
2. $\{t, a$
| $a^{2}=1$,
$\left.a t a^{-1}=t^{-1}\right\}$
3. $\{t, u$
| $\quad u^{4}=1$,
$\left.t^{-1} u t=u\right\}$
4. $\{t, u$
| $u^{8}=1$,
$\left.t^{-1} u t=u\right\}$
5. $\{t, u$
| $\quad u^{8}=1$,
$\left.t^{-1} u t=u^{5}\right\}$
6. $\{t, u, v$
$u^{2}=1$,
$[u, v]=1$
$t^{-1} v t=u v$
$v^{2}=1$,
7. $\left\{t_{1}, t_{2}, a, b, c\right.$
| $\left[t_{1}, t_{2}\right]=1$,
$a^{2}=1$,
$c^{2}=t_{1}$
$a t_{1} a^{-1}=t_{1}^{-1}$
$b t_{1} b^{-1}=t_{2}$,
$t^{-1} u t=u$,
$c t_{1} c^{-1}=t_{1}$,
$b^{2}=a$,
$b^{-1} c^{-1} b c a^{-1}=t_{2}$,
$a t_{2} a^{-1}=t_{2}^{-1}$,
$b t_{2} b^{-1}=t_{1}^{-1}$,
8. $\left\{t_{1}, t_{2}, a, b\right.$
| $\quad\left[t_{1}, t_{2}\right]=1$,
$a^{2}=1$,
$a t_{1} a^{-1}=t_{1}^{-1}$,
$\left.c t_{2} c^{-1}=t_{2}^{-1}\right\}$
$b t_{1} b^{-1}=t_{2}$,
$b^{2}=a$,
$\left.\begin{array}{l}a t_{2} a^{-1}=t_{2}^{-1}, \\ b t_{2} b^{-1}=t_{1}^{-1}\end{array}\right\}$
9. $\{t, a, u$
| $[u, t]=1$,
$u^{2}=1$,
$[u, a]=1$,
ata $\left.^{-1}=t^{-1} u\right\}$
$a^{2}=1$, 


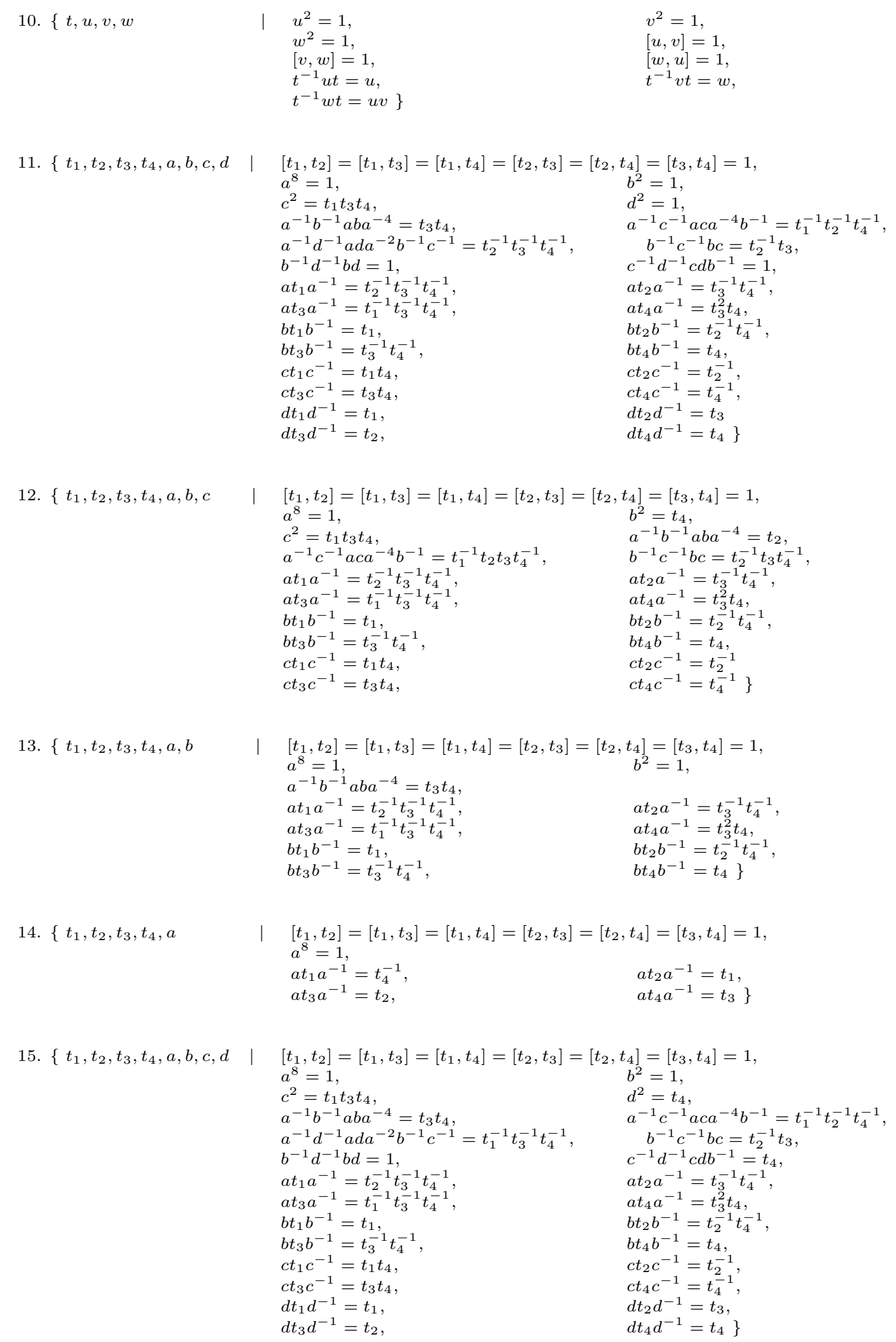

10. $\{t, u, v, w$

$\mid \begin{aligned} & u^{2}=1 \\ & w^{2}=1\end{aligned}$ $w^{2}=1$
$[v, w]=1$ $t^{-1} u t=u$ $\left.t^{-1} w t=u v\right\}$

11. $\left\{t_{1}, t_{2}, t_{3}, t_{4}, a, b, c, d \mid\right.$

$[u, v]=1$,

$[w, u]=1$,

$t^{-1} v t=w$

11. $\left\{t_{1}, t_{2}, t_{3}, t_{4}, a, b, c, d\right\}$$$
a^{8}=1 \text {, }
$$$$
c^{2}
$$$$
c^{2}=t_{1} t_{3} t_{4},
$$$$
a^{-1} b^{-1} a b a^{-4}=t_{3} t_{4} \text {, }
$$$$
a^{-1} d^{-1} a d a^{-2} b^{-1} c^{-1}=t_{2}^{-1} t_{3}^{-1} t_{4}^{-1} \text {, }
$$$$
b^{-1} d^{-1} b d=1 \text {, }
$$$$
a t_{1} a^{-1}=t_{2}^{-1} t_{3}^{-1} t_{4}^{-1} \text {, }
$$$$
a t_{3} a^{-1}=t_{1}^{-1} t_{3}^{-1} t_{4}^{-1} \text {, }
$$$$
b t_{1} b^{-1}=t_{1}
$$$$
b t_{3} b^{-1}=t_{3}^{-1} t_{4}^{-1} \text {, }
$$$$
c t_{1} c^{-1}=t_{1} t_{4}
$$$$
c t_{3} c^{-1}=t_{3} t_{4}
$$$$
d t_{1} d^{-1}=t_{1} \text {, }
$$$$
d t_{3} d^{-1}=t_{2},
$$

$\left.t_{4}\right]=\left[t_{3}, t_{4}\right]=1$,

$b^{2}=1$

$$
d^{2}=1
$$$$
a^{-1} c^{-1} a c a^{-4} b^{-1}=t_{1}^{-1} t_{2}^{-1} t_{4}^{-1} \text {, }
$$$$
b^{-1} c^{-1} b c=t_{2}^{-1} t_{3},
$$$$
c^{-1} d^{-1} c d b^{-1}=1 \text {, }
$$$$
a t_{2} a^{-1}=t_{3}^{-1} t_{4}^{-1} \text {, }
$$$$
a t_{4} a^{-1}=t_{3}^{2} t_{4}
$$$$
b t_{2} b^{-1}=t_{2}^{-1} t_{4}^{-1} \text {, }
$$$$
b t_{4} b^{-1}=t_{4} \text {, }
$$

$c t_{2} c^{-1}=t_{2}^{-1}$,

$c t_{4} c^{-1}=t_{4}^{-1}$,

$d t_{2} d^{-1}=t_{3}$

$\left.d t_{4} d^{-1}=t_{4}\right\}$

12. $\left\{t_{1}, t_{2}, t_{3}, t_{4}, a, b, c\right.$

$$
\begin{array}{lc}
{\left[t_{1}, t_{2}\right]=\left[t_{1}, t_{3}\right]=\left[t_{1}, t_{4}\right]=\left[t_{2}, t_{3}\right]=\left[t_{2}, t_{4}\right]=\left[t_{3}, t_{4}\right]=1,} \\
a^{8}=1, & b^{2}=t_{4}, \\
c^{2}=t_{1} t_{3} t_{4}, & b^{-1} c^{-1} b c=a^{-4}=t_{2}, \\
a^{-1} c^{-1} a c a^{-4} b^{-1}=t_{4}^{-1} & a t_{2} a^{-1}=t_{3}^{-1} t_{4}^{-1}, \\
a t_{1} a^{-1}=t_{2}^{-1} t_{3}^{-1} t_{4}^{-1}, & a t_{4} a^{-1}=t_{3}^{2} t_{4}, \\
a t_{3} a^{-1}=t_{1}^{-1} t_{3}^{-1} t_{4}^{-1}, & b t_{2} b^{-1}=t_{2}^{-1} t_{4}^{-1}, \\
b t_{1} b^{-1}=t_{1}, & b t_{4} b^{-1}=t_{4}, \\
b t_{3} b^{-1}=t_{3}^{-1} t_{4}^{-1}, & c t_{2} c^{-1}=t_{2}^{-1} \\
c t_{1} c^{-1}=t_{1} t_{4}, & \left.c t_{4} c^{-1}=t_{4}^{-1}\right\} \\
c t_{3} c^{-1}=t_{3} t_{4}, &
\end{array}
$$

13. $\left\{t_{1}, t_{2}, t_{3}, t_{4}, a, b\right.$

$$
\begin{aligned}
& a^{8}=1, \\
& a^{-1} b^{-1} a b a^{-4}=t_{3} t_{4}, \\
& a t_{1} a^{-1}=t_{2}^{-1} t_{3}^{-1} t_{4}^{-1}, \\
& a t_{3} a^{-1}=t_{1}^{-1} t_{3}^{-1} t_{4}^{-1}, \\
& b t_{1} b^{-1}=t_{1} \\
& b t_{3} b^{-1}=t_{3}^{-1} t_{4}^{-1},
\end{aligned}
$$$$
\left.t_{4}\right]=\left[t_{3}, t_{4}\right]=1 \text {, }
$$$$
b^{2}=1 \text {, }
$$$$
a t_{2} a^{-1}=t_{3}^{-1} t_{4}^{-1},
$$$$
a t_{4} a^{-1}=t_{3}^{2} t_{4}
$$$$
b t_{2} b^{-1}=t_{2}^{-1} t_{4}^{-1},
$$$$
\left.b t_{4} b^{-1}=t_{4}\right\}
$$

14. $\left\{t_{1}, t_{2}, t_{3}, t_{4}, a\right.$

| $\left[t_{1}, t_{2}\right]=\left[t_{1}, t_{3}\right]=\left[t_{1}, t_{4}\right]=\left[t_{2}, t_{3}\right]=\left[t_{2}, t_{4}\right]=\left[t_{3}, t_{4}\right]=1$,

$$
\left.\begin{array}{ll}
a^{8}=1, & \\
a t_{1} a^{-1}=t_{4}^{-1}, & a t_{2} a^{-1}=t_{1}, \\
a t_{3} a^{-1}=t_{2}, & a t_{4} a^{-1}=t_{3}
\end{array}\right\}
$$$$
\left.a t_{4} a^{-1}=t_{3}\right\}
$$

15. $\left\{t_{1}, t_{2}, t_{3}, t_{4}, a, b, c, d \quad \mid\right.$

$$
\begin{aligned}
& a^{8}=1, \\
& c^{2}=t_{1} t_{3} t_{4}, \\
& a^{-1} b^{-1} a b a^{-4}=t_{3} t_{4}, \\
& a^{-1} d^{-1} a d a^{-2} b^{-1} c^{-1}=t_{1}^{-1} t_{3}^{-1} t_{4}^{-1}, \\
& b^{-1} d^{-1} b d=1, \\
& a t_{1} a^{-1}=t_{2}^{-1} t_{3}^{-1} t_{4}^{-1}, \\
& a t_{3} a^{-1}=t_{1}^{-1} t_{3}^{-1} t_{4}^{-1}, \\
& b t_{1} b^{-1}=t_{1}, \\
& b t_{3} b^{-1}=t_{3}^{-1} t_{4}^{-1}, \\
& c t_{1} c^{-1}=t_{1} t_{4}, \\
& c t_{3} c^{-1}=t_{3} t_{4}, \\
& d t_{1} d^{-1}=t_{1}, \\
& d t_{3} d^{-1}=t_{2},
\end{aligned}
$$$$
\left[t_{1}, t_{2}\right]=\left[t_{1}, t_{3}\right]=\left[t_{1}, t_{4}\right]=\left[t_{2}, t_{3}\right]=\left[t_{2}, t_{4}\right]=\left[t_{3}, t_{4}\right]=1 \text {, }
$$$$
b^{2}=1 \text {, }
$$$$
d^{2}=t_{4},
$$$$
a^{-1} c^{-1} a c a^{-4} b^{-1}=t_{1}^{-1} t_{2}^{-1} t_{4}^{-1} \text {, }
$$$$
b^{-1} c^{-1} b c=t_{2}^{-1} t_{3}
$$$$
c^{-1} d^{-1} c d b^{-1}=t_{4} \text {, }
$$$$
a t_{2} a^{-1}=t_{3}^{-1} t_{4}^{-1} \text {, }
$$$$
a t_{4} a^{-1}=t_{3}^{2} t_{4} \text {, }
$$$$
\begin{aligned}
& a t_{4} a=t_{2} b^{-1}=t_{2}^{-1} t_{4}^{-1}, \\
& b t_{4} b^{-1}=
\end{aligned}
$$$$
b t_{4} b^{-1}=t_{4}
$$$$
c t_{2} c^{-1}=t_{2}^{-1}
$$$$
c t_{4} c^{-1}=t_{4}^{-1} \text {, }
$$$$
d t_{2} d^{-1}=t_{3},
$$$$
\left.d t_{4} d^{-1}=t_{4}\right\}
$$

$v^{2}=1$, 
16. $\left\{t_{1}, t_{2}, t_{3}, t_{4}, a, b, c, d\right.$

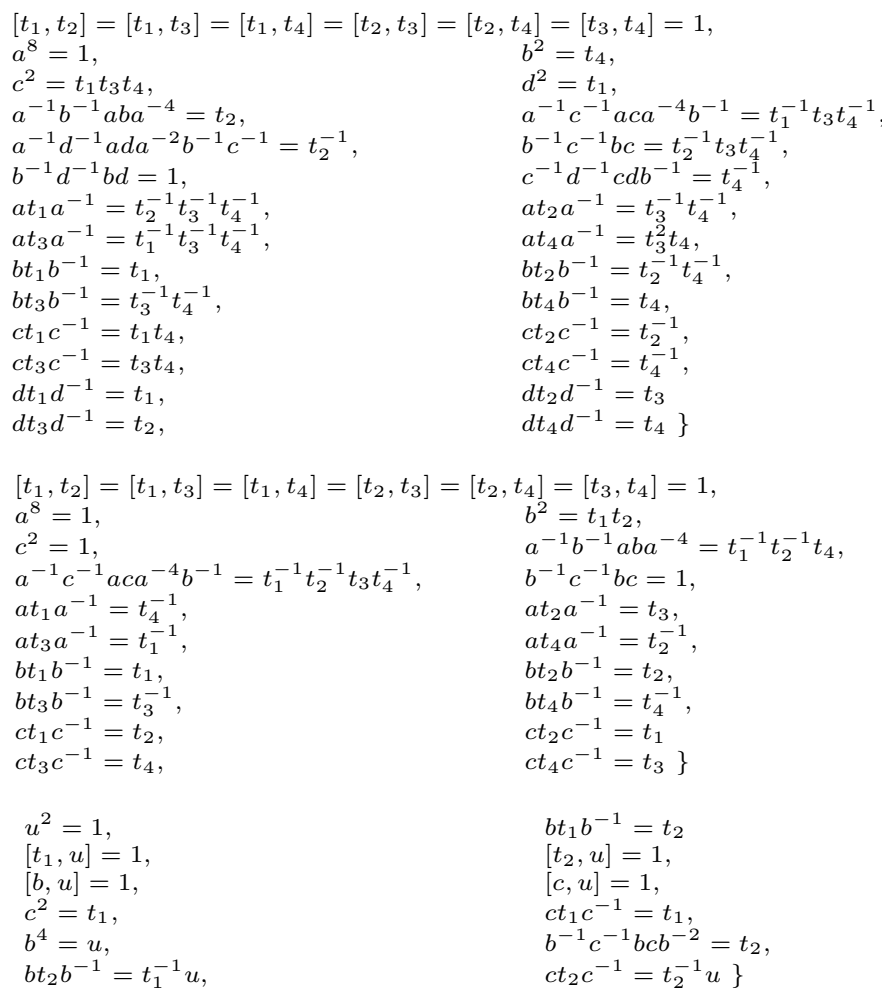
$\left[t_{1}, t_{2}\right]$
$a^{8}=1$ $c^{2}=1$,

$a^{-1} c^{-1} a c a^{-4} b^{-1}=t_{1}^{-1} t_{2}^{-1} t_{3} t_{4}^{-1}$, $a t_{1} a^{-1}=t_{4}^{-1}$,

$a t_{3} a^{-1}=t_{1}^{-1}$,

$b t_{1} b^{-1}=t_{1}$,

$b t_{3} b^{-1}=t_{3}^{-1}$,

$c t_{1} c^{-1}=t_{2}$,

$\left.t_{4}\right]=\left[t_{3}, t_{4}\right]=1$,

$c t_{3} c^{-1}=t_{4}$,

$b^{2}=t_{1} t_{2}$

$a^{-1} b^{-1} a b a^{-4}=t_{1}^{-1} t_{2}^{-1} t_{4}$,

$b^{-1} c^{-1} b c=1$,

$a t_{2} a^{-1}=t_{3}$,

$a t_{4} a^{-1}=t_{2}^{-1}$,

$b t_{2} b^{-1}=t_{2}$,

$b t_{4} b^{-1}=t_{4}^{-1}$,

$c t_{2} c^{-1}=t_{1}$

$\left[t_{1}, u\right]=1$,

$[b, u]=1$,

$c^{2}=t_{1}$,

$b^{4}=u$,

$b t_{2} b^{-1}=t_{1}^{-1} u$,

$\left.c t_{4} c^{-1}=t_{3}\right\}$

$b t_{1} b^{-1}=t_{2}$

$\left[t_{2}, u\right]=1$,

$[c, u]=1$,

$c t_{1} c^{-1}=t_{1}$,

$b^{-1} c^{-1} b c b^{-2}=t_{2}$,

$\left.c t_{2} c^{-1}=t_{2}^{-1} u\right\}$

17. $\left\{t_{1}, t_{2}, t_{3}, t_{4}, a, b, c\right.$

I $\quad\left[t_{1}\right.$

18. $\left\{t_{1}, t_{2}, b, c, u\right.$

| $u^{2}=1$,

19. $\left\{t_{1}, t_{2}, t_{3}, t_{4}, a, b\right.$
$\left[t_{1}, t_{2}\right]=$
$a^{8}=1$ $a^{-1} b^{-1} a b a^{-4}=t_{1}^{-1} t_{2}^{-1} t_{3}$, $a t_{1} a^{-1}=t_{3}^{-1}$, $a t_{3} a^{-1}=t_{2}^{-1}$, $b t_{1} b^{-1}=t_{1}$
$b t_{3} b^{-1}=t_{3}^{-1}$,

$\begin{aligned}\left., t_{4}\right] & =\left[t_{3}, t_{4}\right]=1, \\ b^{2} & =t_{1} t_{2},\end{aligned}$

$a t_{2} a^{-1}=t_{4}^{-1}$

$a t_{4} a^{-1}=t_{1}$,

$b t_{2} b^{-1}=t_{2}$

$\left.b t_{4} b^{-1}=t_{4}^{-1}\right\}$

20. $\left\{t_{1}, t_{2}, t_{3}, t_{4}, a, b, c\right.$

$$
\begin{aligned}
& {\left[t_{1}, t_{2}\right]=\left[t_{1}, t_{3}\right]=\left[t_{1}, t_{4}\right]=} \\
& a^{8}=1 \\
& c^{2}=t_{1} t_{3} t_{4} \\
& a^{-1} c^{-1} a c a^{-4} b^{-1}=t_{1}^{-1} t_{4}^{-1} \\
& a t_{1} a^{-1}=t_{2}^{-1} t_{3}^{-1} t_{4}^{-1} \\
& a t_{3} a^{-1}=t_{1}^{-1} t_{3}^{-1} t_{4}^{-1} \\
& b t_{1} b^{-1}=t_{1}, \\
& b t_{3} b^{-1}=t_{3}^{-1} t_{4}^{-1} \\
& c t_{1} c^{-1}=t_{1} t_{4} \\
& c t_{3} c^{-1}=t_{3} t_{4}
\end{aligned}
$$

$$
\begin{aligned}
& \left.t_{4}\right]=\left[t_{3}, t_{4}\right]=1, \\
& b^{2}=1, \\
& a^{-1} b^{-1} a b a^{-4}=t_{3} t_{4}, \\
& b^{-1} c^{-1} b c=t_{2}^{-1} t_{3}, \\
& a t_{2} a^{-1}=t_{3}^{-1} t_{4}^{-1}, \\
& a t_{4} a^{-1}=t_{3}^{2} t_{4}, \\
& b t_{2} b^{-1}=t_{2}^{-1} t_{4}^{-1} \\
& b t_{4} b^{-1}=t_{4} \\
& c t_{2} c^{-1}=t_{2}^{-1} \\
& c t_{4} c^{-1}=t_{4}^{-1}
\end{aligned}
$$

21. $\left\{t_{1}, t_{2}, b, u\right.$

| $u^{2}=1$

$$
\begin{aligned}
& {\left[t_{1}, u\right]=1,} \\
& b^{-1} t_{1} b=t_{2}, \\
& b^{4}=u,
\end{aligned}
$$

22. $\{t, u, v$

23. $\left\{t_{1}, t_{2}, b, u\right.$

| $\quad u^{4}=1$,

$$
\begin{aligned}
& {[u, v]=1,} \\
& \left.t^{-1} v t=u^{2} v\right\}
\end{aligned}
$$

$[b, u]=1$

$\left[t_{2}, u\right]=1$,

$\left[t_{1}, t_{2}\right]=1$,

$\left.b^{-1} t_{2} b=t_{1}^{-1} u\right\}$

$v^{2}=1$,

$t^{-1} u t=u v$,

$$
\left.\begin{array}{l}
{[b, u]=1} \\
{\left[t_{2}, u\right]=1,} \\
{\left[t_{1}, t_{2}\right]=1} \\
b^{-1} t_{2} b=t_{1}^{-1}
\end{array}\right\}
$$


31. $\left\{t_{1}, t_{2}, t_{3}, t_{4}, a, b, c \quad \mid\right.$

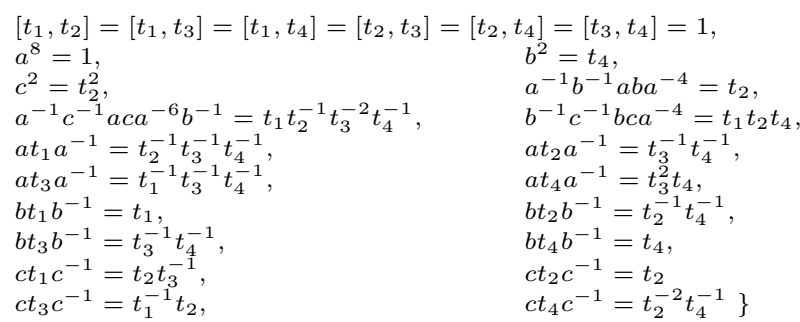

32. $\left\{t_{1}, t_{2}, t_{3}, t_{4}, a, b\right.$

| $\quad\left[t_{1}, t_{2}\right]=\left[t_{1}, t_{3}\right]=\left[t_{1}, t_{4}\right]=\left[t_{2}, t_{3}\right]=\left[t_{2}, t_{4}\right]=\left[t_{3}, t_{4}\right]=1$, $a^{8}=1$, $a^{-1} b^{-1} a b a^{6}=t_{2}^{2} t_{3}^{-1}$

33. $\left\{t_{1}, t_{2}, t_{3}, t_{4}, a, b \quad \mid \quad\left[t_{1}, t_{2}\right]=\left[t_{1}, t_{3}\right]=\left[t_{1}, t_{4}\right]=\left[t_{2}, t_{3}\right]=\left[t_{2}, t_{4}\right]=\left[t_{3}, t_{4}\right]=1\right.$, $a^{8}=1, \quad b^{2}=1$

34. $\left\{t_{1}, t_{2}, t_{3}, t_{4}, a, b, c \mid\right.$

$\left[t_{1}, t_{2}\right]=\left[t_{1}, t_{3}\right]=\left[t_{1}, t_{4}\right]=\left[t_{2}, t_{3}\right]=\left[t_{2}, t_{4}\right]=\left[t_{3}, t_{4}\right]=1$,

$a^{4}=1$,

$b^{4}=t_{1}^{2} t_{4}^{2}$,

$c^{4}=t_{1} t_{2} t_{4}$

$a^{-1} c^{-1} a c a^{-2} b^{-2}=t_{1}^{-1} t_{2} t_{3}^{-2} t_{4}^{-1}$,

$a^{-1} b^{-1} a b=t_{1} t_{3}^{-1}$

$a t_{1} a^{-1}=t_{2}^{-1} t_{3}$,

$a t_{3} a^{-1}=t_{3} t_{4}$

$b t_{1} b^{-1}=t_{2}^{-1} t_{3}$

$b t_{3} b^{-1}=t_{1}^{-1} t_{3}$,

$c t_{1} c^{-1}=t_{1} t_{4}$,

$c t_{3} c^{-1}=t_{1} t_{2}^{-1}$,

$b^{-1} c^{-1} b c a^{-3}=t_{1}^{-1} t_{4}^{-1}$,

$a t_{2} a^{-1}=t_{1} t_{3} t_{4}$,

$a_{4} a^{-1}=t_{3}^{-2} t_{4}^{-1}$

$b t_{2} b^{-1}=t_{3}$,

$b t_{4} b^{-1}=t_{1} t_{2} t_{3}^{-1} t_{4}$,

$c t_{2} c^{-1}=t_{1} t_{3} t_{4}$,

$\left.c t_{4} c^{-1}=t_{1}^{-1} t_{2} t_{3}^{-1} t_{4}^{-1}\right\}$

35. $\left\{t_{1}, t_{2}, t_{3}, t_{4}, a, b, c, d\right.$

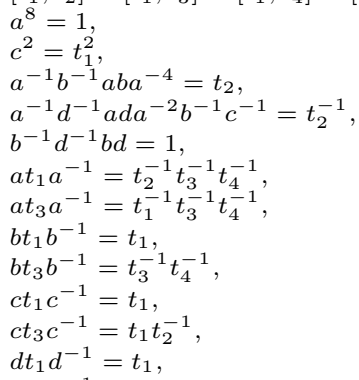

36. $\left\{t_{1}, t_{2}, b, u\right.$

$u^{2}=1$,

$\left[t_{1}, u\right]=1$, $b^{-1} t_{1} b=t_{2}$, $b^{4}=1$,

$[b, u]=1$

$\left[t_{2}, u\right]=1$,

$\left[t_{1}, t_{2}\right]=u$

$\left.b^{-1} t_{2} b=t_{1}^{-1}\right\}$

37. $\left\{t_{1}, t_{2}, b, c, u\right.$

| $u^{2}=1$,

$\left[t_{1}, u\right]=1$,
$[b, u]=1$,

$c^{2}=t_{1}$,

$b^{4}=1$,

$b t_{2} b^{-1}=t_{1}^{-1} u$,

$b t_{1} b^{-1}=t_{2}$

$\left[t_{2}, u\right]=1$,

$[c, u]=1$,

$c t_{1} c^{-1}=t_{1}$,
$b^{-1} c^{-1} b c b^{-2}=t_{2} u$

$\left.c t_{2} c^{-1}=t_{2}^{-1} u\right\}$ 
38. $\left\{t_{1}, t_{2}, b, c, u\right.$

39. $\{t, a, u, v$ $u^{2}=1$,

$$
\left[t_{1}, u\right]=1 \text {, }
$$

$[b, u]=1$,

$c^{2}=t_{1}$,

$b^{4}=u$,

$b t_{2} b^{-1}=t_{1}^{-1}$,

| $u^{2}=1$,

$[u, v]=1$,

$a^{-1} u a=u$,

$t^{-1} u t=v$,

$\left.a^{2}=u\right\}$

$$
\begin{aligned}
& b t_{1} b^{-1}=t_{2} \\
& {\left[t_{2}, u\right]=1} \\
& {[c, u]=1} \\
& c t_{1} c^{-1}=t_{1} \\
& b^{-1} c^{-1} b c b^{-2}=t_{2} u \\
& \left.c t_{2} c^{-1}=t_{2}^{-1}\right\}
\end{aligned}
$$

$v^{2}=1$,

$a^{-1} t a=t^{-1} u$,

$a^{-1} v a=v$,

$t^{-1} v t=u$,

40. $\left\{t_{1}, t_{2}, t_{3}, t_{4}, a, b, c, d \mid\right.$

$\begin{array}{ll}{\left[t_{1}, t_{2}\right]=\left[t_{1}, t_{3}\right]=\left[t_{1}, t_{4}\right]=\left[t_{2}, t_{3}\right]=\left[t_{2}, t_{4}\right]=\left[t_{3}, t_{4}\right]=1,} \\ a^{8}=1, & b^{2}=t_{3} t_{4}, \\ c^{2}=1, & d^{2}=t_{4}, \\ a^{-1} b^{-1} a b a^{-4}=t_{1}^{-1} t_{3}^{-1} t_{4}^{-1}, & a^{-1} c^{-1} a c a^{-4} b^{-1}=t_{1} t_{2}^{-1} t_{3}^{-1} t_{4}^{-1}, \\ a^{-1} d^{-1} a d a^{-2} b^{-1} c^{-1}=t_{3}^{-1}, & b^{-1} c^{-1} b c=1, \\ b^{-1} d^{-1} b d=1, & c^{-1} d^{-1} c d b^{-1}=t_{3}^{-1}, \\ a t_{1} a^{-1}=t_{3}, & a t_{2} a^{-1}=t_{4}, \\ a t_{3} a^{-1}=t_{2}^{-1}, & a t_{4} a^{-1}=t_{1}, \\ b t_{1} b^{-1}=t_{1}^{-1}, & b t_{2} b^{-1}=t_{2}^{-1}, \\ b t_{3} b^{-1}=t_{3}, & b t_{4} b^{-1}=t_{4}, \\ c t_{1} c^{-1}=t_{2}, & c t_{2} c^{-1}=t_{1}, \\ c t_{3} c^{-1}=t_{4}, & c t_{4} c^{-1}=t_{3}, \\ d t_{1} d^{-1}=t_{1}, & d t_{2} d^{-1}=t_{2}^{-1} \\ d t_{3} d^{-1}=t_{3}, & \left.d t_{4} d^{-1}=t_{4}\right\}\end{array}$

41. $\left\{t_{1}, t_{2}, t_{3}, t_{4}, a, b, c\right.$$$
[t
$$$$
\left[t_{1}, t_{2}\right]=\left[t_{1}, t_{3}\right]
$$$$
a^{8}=1 \text {, }
$$$$
\begin{aligned}
& c^{2}=t_{2}, \\
& a^{-1} c^{-1} a c a^{-6} b^{-1}=t_{2}^{-1} t_{3}^{-1} t_{4}^{-1},
\end{aligned}
$$$$
a t_{1} a^{-1}=t_{2}^{-1} t_{3}^{-1} t_{4}^{-1} \text {, }
$$$$
a t_{3} a^{-1}=t_{1}^{-1} t_{3}^{-1} t_{4}^{-1},
$$$$
b t_{1} b^{-1}=t_{1} \text {, }
$$$$
b t_{3} b^{-1}=t_{3}^{-1} t_{4}^{-1},
$$$$
c t_{1} c^{-1}=t_{2} t_{3}^{-1} \text {, }
$$$$
c t_{3} c^{-1}=t_{1}^{-1} t_{2} \text {, }
$$

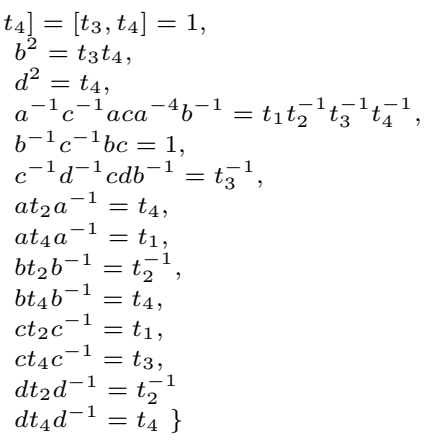$$
\left.d t_{4} d^{-1}=t_{4}\right\}
$$

42. The pro-2-group for this family has point group $Q_{16}$. See discussion in Section 2 .

43. $\left\{t_{1}, t_{2}, t_{3}, t_{4}, a, b\right.$

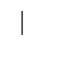

$$
\begin{aligned}
& a^{8}=1 \\
& a^{-1} b^{-1} a b a^{6}=t_{1}^{-1} t_{2} t_{3}^{-1}, \\
& a t_{1} a^{-1}=t_{4}, \\
& a t_{3} a^{-1}=t_{1}, \\
& b t_{1} b^{-1}=t_{2}^{-1} \\
& b t_{3} b^{-1}=t_{3}^{-1}
\end{aligned}
$$

$$
\left.\begin{array}{l}
\left.t_{4}\right]=\left[t_{3}, t_{4}\right]=1, \\
b^{2}=t_{4} \\
a t_{2} a^{-1}=t_{3}^{-1} \\
a t_{4} a^{-1}=t_{2} \\
b t_{2} b^{-1}=t_{1}^{-1} \\
b t_{4} b^{-1}=t_{4}
\end{array}\right\}
$$

44. $\left\{t_{1}, t_{2}, b, c, u\right.$

| $u^{2}=1$,

$\left[t_{1}, u\right]=1$
$[b, u]=1$

$c^{2}=t_{1}$,

$b^{4}=1$

$b t_{2} b^{-1}=t_{1}^{-1} u$

$b t_{1} b^{-1}=t_{2}$

$\left[t_{2}, u\right]=1$,

$[c, u]=1$,

$c t_{1} c^{-1}=t_{1}$,
$b^{-1} c^{-1} b c b^{-2}=t_{2}$,

$\left.c t_{2} c^{-1}=t_{2}^{-1} u\right\}$

45. $\left\{t_{1}, t_{2}, b, u\right.$

| $u^{2}=1$

$\left[t_{1}, u\right]=1$,
$b^{-1} t_{1} b=t_{2}$ $b^{4}=u$,

$[b, u]=1$

$\left[t_{2}, u\right]=1$,

$\left[t_{1}, t_{2}\right]=u$

$\left.b^{-1} t_{2} b=t_{1}^{-1}\right\}$ 

46. $\left\{t_{1}, t_{2}, b, c, u\right.$
$\mid u^{2}=1$,
$\left[t_{1}, u\right]=1$,
$[b, u]=1$,
$c^{2}=t_{1}$,
$b^{4}=u$,
$b t_{2} b^{-1}=t_{1}^{-1}$,
$b t_{1} b^{-1}=t_{2}$
$\left[t_{2}, u\right]=1$
$[c, u]=1$
$c t_{1} c^{-1}=t_{1}$
$b^{-1} c^{-1} b c b^{-2}=t_{2}$
$\left.c t_{2} c^{-1}=t_{2}^{-1}\right\}$
47. $\{t, a, u$
| $u^{4}=1$,
$t^{-1} u t=u^{-1}$,
$\left.a^{2}=u\right\}$
$a^{-1} t a=t^{-1} u$
$a^{-1} u a=u$,
48. $\{t, a, u$
| $u^{4}=1$,
$t^{-1} u t=u^{-1}$
$a^{-1} t a=t^{-1} u$,
$\left.a^{2}=u^{-1}\right\}$
$a^{-1} u a=u$,
49. $\{t, u$
| $u^{4}=1$,
$\left.t^{-1} u t=u^{-1}\right\}$
50. $\{t, a, u$
| $[u, t]=1$,
$\left.\begin{array}{l}u^{2}=1, \\ a t a^{-1}=t^{-1}\end{array}\right\}$
$[u, a]=1$,
51. $\{t, u$
| $u^{8}=1$,
$\left.t^{-1} u t=u^{3}\right\}$
52. $\{t, u$
| $u^{8}=1$,
$\left.t^{-1} u t=u^{-1}\right\}$
53. $\{t, a, u$
| $u^{4}=1$,
$t^{-1} u t=u$
$a^{-1} t a=t^{-1}$,
$\left.a^{2}=u\right\}$
54. $\{t, u, v$
| $u^{4}=1$,
$[u, v]=1$,
$v^{2}=1$,
$t^{-1} u t=u v$
$\left.t^{-1} v t=v\right\}$
55. $\{t, a, u, v$
| $u^{2}=1$,
$[u, v]=1$,
$a^{-1} u a=u$,
$t^{-1} u t=u$,
$\left.a^{2}=v\right\}$
56. $\left\{t_{1}, t_{2}, b, c, u\right.$
| $u^{2}=1$,
$\left[t_{1}, u\right]=1$,
$[b, u]=1$,
$c^{2}=t_{1}$,
$b^{4}=1$,
$b^{4}=1$,
$b t_{2} b^{-1}=t_{1}^{-1}$,
$a^{-1} u a=u$,
57. $\left\{t_{1}, t_{2}, b, u\right.$
| $u^{2}=1$
$\left[t_{1}, u\right]=1$,
$b^{-1} t_{1} b=t_{2}$ $b^{-1} t_{1} b$
$b^{4}=1$
$v^{2}=1$,
$v^{2}=1$,
$a^{-1} t a=t^{-1} u$,
$a^{-1} v a=v$
$t^{-1} v t=v$,
$b t_{1} b^{-1}=t_{2}$
$\left[t_{2}, u\right]=1$,
$[c, u]=1$,
$c t_{1} c^{-1}=t_{1}$,
$b^{-1} c^{-1} b c b^{-2}=t_{2} u$
$\left.c t_{2} c^{-1}=t_{2}^{-1}\right\}$
$[b, u]=1$
$\left[t_{2}, u\right]=1$,
$\left[t_{1}, t_{2}\right]=1$,
$\left.b^{-1} t_{2} b=t_{1}^{-1} u\right\}$
58. $\{t, u, v$
| $u^{2}=1$,
$[u, v]=1$,
$v^{2}=1$,
$t^{-1} u t=u$,
59. $\{t, a, u$
| $\quad[u, t]=1$,
$u^{2}=1$,
ata $\left.^{-1}=t^{-1}\right\}$
$[u, a]=1$,
$a^{2}=1$,
60. $\{t, u, v$
| $u^{4}=1$,
$[u, v]=1$,
$\left.t^{-1} v t=v\right\}$
$v^{2}=1$,
$t^{-1} u t=u$, 
61. $\{t, u, v, w$

62. $\left\{t_{1}, t_{2}, b, c, u\right.$

63. $\left\{t_{1}, t_{2}, b, u\right.$

64. $\{t, a, u, v$

65. $\{t, u, v$

66. $\{t, a, u, v$

67. $\{t, u, v$

68. $\{t, u, v$

69. $\{t, a, u$

70. $\{t, u, v$

71. $\{t, a, u, v$

72. $\left\{t_{1}, t_{2}, a, b, c\right.$

73. $\{t, a, u$
M. F. NEWMAN AND E. A. O'BRIEN

| $u^{2}=1$

$w^{2}=1$

$[v, w]=1$,

$t^{-1} u t=u$,

$\left.t^{-1} w t=u w\right\}$

| $u^{2}=1$,

$\left[t_{1}, u\right]=1$,

$[b, u]=1$,

$c^{2}=t_{1}$,

$b^{4}=1$,

$b t_{2} b^{-1}=t_{1}^{-1}$,

| $u^{2}=1$

$\left[t_{1}, u\right]=1$,

$b^{-1} t_{1} b=t_{2}$,

$b^{4}=1$,

| $u^{2}=1$,

$[u, v]=1$,

$a^{-1} u a=u$,

$t^{-1} u t=u$,

$\left.a^{2}=1\right\}$

| $u^{4}=1$,

$[u, v]=1$,

$\left.t^{-1} v t=v\right\}$

| $u^{2}=1$,

$[u, v]=1$,

$a^{-1} u a=u$

$t^{-1} u t=u$

$\left.a^{2}=u\right\}$

| $u^{4}=1$,

$[u, v]=1$,

$\left.t^{-1} v t=u^{2} v\right\}$

| $u^{4}=1$,

$[u, v]=u^{2}$,
$\left.t^{-1} v t=v\right\}$

| $u^{4}=1$,

$t^{-1} u t=u$,

$\left.a^{2}=1\right\}$

| $u^{4}=1$,

$\left.\begin{array}{l}{[u, v]=u^{2},} \\ t^{-1} v t=v\end{array}\right\}$

| $u^{2}=1$,

$[u, v]=1$,

$a^{-1} u a=u$,

$t^{-1} u t=v$,

$\left.a^{2}=1\right\}$

| $\left[t_{1}, t_{2}\right]=1$,

$a^{2}=1$,
$c^{2}=1$,

$a t_{1} a^{-1}=t_{1}^{-1}$,

$b t_{1} b^{-1}=t_{2}$,

$c t_{1} c^{-1}=t_{1}$,

| $u^{4}=1$,

$t^{-1} u t=u^{-1}$,

$\left.a^{2}=1\right\}$ $v^{2}=1$,

$[u, v]=1$

$[w, u]=1$,

$b t_{1} b^{-1}=t_{2}$

$\left[t_{2}, u\right]=1$,

$[c, u]=1$,

$c t_{1} c^{-1}=t_{1}$,

$b^{-1} c^{-1} b c b^{-2}=t_{2}$

$\left.c t_{2} c^{-1}=t_{2}^{-1}\right\}$

$[b, u]=1$

$\left[t_{2}, u\right]=1$,

$\left[t_{1}, t_{2}\right]=1$

$\left.b^{-1} t_{2} b=t_{1}^{-1}\right\}$

$v^{2}=1$,

$a^{-1} t a=t^{-1} u$,

$a^{-1} v a=v$

$t^{-1} v t=v$,

$v^{2}=1$,

$t^{-1} u t=u^{-1}$,

$v^{2}=1$,

$a^{-1} t a=t^{-1}$,

$a^{-1} v a=v$

$t^{-1} v t=v$

$v^{2}=1$,

$t^{-1} u t=u$,

$v^{2}=1$,

$t^{-1} u t=u$,

$a^{-1} t a=t^{-1}$,

$a^{-1} u a=u$,

$v^{2}=u^{2}$,

$t^{-1} u t=u$,

$v^{2}=1$,

$a^{-1} t a=t^{-1}$,

$a^{-1} v a=v$,

$t^{-1} v t=u$,

$b^{2}=a$,

$[b, c]=a$,

$a t_{2} a^{-1}=t_{2}^{-1}$,

$b t_{2} b^{-1}=t_{1}^{-1}$,

$\left.c t_{2} c^{-1}=t_{2}^{-1}\right\}$

$a^{-1} t a=t^{-1}$,

$a^{-1} u a=u$, 


\begin{tabular}{|c|c|c|}
\hline 74. $\{t, a, u, v$ & $\begin{array}{l}u^{2}=1, \\
\quad[u, v]=1, \\
a^{-1} u a=v, \\
t^{-1} u t=u \\
\left.a^{2}=1\right\}\end{array}$ & $\begin{array}{l}v^{2}=1 \\
a^{-1} t a=t^{-1} \\
a^{-1} v a=u \\
t^{-1} v t=v\end{array}$ \\
\hline 75. $\{t, a, u$ & $\begin{array}{l}u^{4}=1 \\
t^{-1} u t=u^{-1} \\
\left.a^{2}=u^{2}\right\}\end{array}$ & $\begin{array}{l}a^{-1} t a=t^{-1} \\
a^{-1} u a=u\end{array}$ \\
\hline 76. $\{t, a, u, v$ & $\begin{array}{l}u^{2}=1, \\
{[u, v]=1,} \\
a^{-1} u a=u, \\
t^{-1} u t=v, \\
\left.a^{2}=u v\right\}\end{array}$ & $\begin{array}{l}v^{2}=1 \\
a^{-1} t a=t^{-1} \\
a^{-1} v a=v \\
t^{-1} v t=u\end{array}$ \\
\hline 77. $\{t, a, u$ & $\mid \begin{array}{l}u^{4}=1 \\
t^{-1} u t=u \\
\left.a^{2}=1\right\}\end{array}$ & $\begin{array}{l}a^{-1} t a=t^{-1} u^{2} \\
a^{-1} u a=u^{-1}\end{array}$ \\
\hline 78. $\{t, a, u$ & $\begin{array}{l}u^{4}=1 \\
t^{-1} u t=u \\
\left.a^{2}=1\right\}\end{array}$ & $\begin{array}{l}a^{-1} t a=t^{-1} \\
a^{-1} u a=u^{-1}\end{array}$ \\
\hline 79. $\{t, a, u$ & $\begin{array}{l}u^{4}=1 \\
t^{-1} u t=u \\
\left.a^{2}=u^{2}\right\}\end{array}$ & $\begin{array}{l}a^{-1} t a=t^{-1} \\
a^{-1} u a=u^{-1}\end{array}$ \\
\hline 80. $\{t, u, v, w$ & $\begin{array}{l}u^{2}=1 \\
w^{2}=1 \\
{[v, w]=1} \\
t^{-1} u t=u \\
\left.t^{-1} w t=w\right\}\end{array}$ & $\begin{array}{l}v^{2}=1 \\
{[u, v]=1} \\
{[w, u]=1} \\
t^{-1} v t=v\end{array}$ \\
\hline 81. $\{t, a, u, v$ & $\begin{array}{l}u^{2}=1, \\
{[u, v]=1,} \\
\\
a^{-1} u a=u \\
t^{-1} u t=u \\
\left.a^{2}=1\right\}\end{array}$ & $\begin{array}{l}v^{2}=1 \\
a^{-1} t a=t^{-1} \\
a^{-1} v a=v \\
t^{-1} v t=v\end{array}$ \\
\hline
\end{tabular}

Appendix B. The families of 2-groups of Coclass at most 3

We now summarise the descendant patterns for the 12 families of 2-groups of coclass at most 2 and the conjectured patterns for the 70 families of 2-groups of coclass 3. The summary tables follow the conventions introduced in Section 5 . The tables are taken to begin at the periodic root. A • at level $2^{n+k}$ indicates that the (conjectured) period is $k$; otherwise it is the number of levels in the table. If the shortest path in $\mathcal{F}_{G}$ between a terminal group $G$ and a mainline group has length $t$, then $\mathcal{F}_{G}$ has a twig of length $t$. We record the (conjectured) largest twig length for each family.

\begin{tabular}{|c|r|}
\hline Order & Structure \\
\hline $2^{n+1}$ & $2 / 1$ \\
\hline
\end{tabular}

TABLE 0 . Family \#0 with periodic root order $2^{1} \&$ twig length 1

\begin{tabular}{|c|r|}
\hline Order & Structure \\
\hline $2^{n+1}$ & $2 / 1$ \\
\hline
\end{tabular}

TABLE 1 . Family \#1 with periodic root order $2^{3} \&$ twig length 1 


\begin{tabular}{|c|r|}
\hline Order & Structure \\
\hline $2^{n+1}$ & $3 / 1$ \\
\hline
\end{tabular}

TABle 2. Family \#2 with periodic root order $2^{3} \&$ twig length 1

\begin{tabular}{|c|r|r|}
\hline Order & \multicolumn{2}{|c|}{ Structure } \\
\hline $2^{n+1}$ & $2 / 2$ & $\bullet$ \\
\hline $2^{n+2}$ & 1 & \\
\hline
\end{tabular}

TABLE 3 . Family \#3 with periodic root order $2^{5} \&$ twig length 2

\begin{tabular}{|c|r|r|}
\hline Order & \multicolumn{2}{|c|}{ Structure } \\
\hline $2^{n+1}$ & $2 / 2$ & $\bullet$ \\
\hline $2^{n+2}$ & $1 / 1$ & \\
\hline $2^{n+3}$ & 1 & \\
\hline
\end{tabular}

TABLE 4 . Family \#4 with periodic root order $2^{7} \&$ twig length 3

\begin{tabular}{|c|r|r|}
\hline Order & \multicolumn{2}{|c|}{ Structure } \\
\hline $2^{n+1}$ & $2 / 2$ & $\bullet$ \\
\hline $2^{n+2}$ & 1 & \\
\hline
\end{tabular}

TABLE 5 . Family \#5 with periodic root order $2^{7} \&$ twig length 2

\begin{tabular}{|c|r|}
\hline Order & Structure \\
\hline $2^{n+1}$ & $3 / 1$ \\
\hline
\end{tabular}

TABLE 6 . Family \#6 with periodic root order $2^{5} \&$ twig length 1

\begin{tabular}{|c|l|r|}
\hline Order & \multicolumn{2}{|c|}{ Structure } \\
\hline $2^{n+1}$ & $4 / 2$ & \\
\hline $2^{n+2}$ & $8 / 1$ & 4 \\
\hline
\end{tabular}

TABLE 7 . Family \# 7 with periodic root order $2^{6} \&$ twig length 2

\begin{tabular}{|c|r|r|r|}
\hline Order & \multicolumn{3}{|c|}{ Structure } \\
\hline $2^{n+1}$ & $6 / 2$ & & \\
\hline $2^{n+2}$ & $6 / 2$ & 4 & $\bullet$ \\
\hline $2^{n+3}$ & 3 & & \\
\hline
\end{tabular}

TABLE 8 . Family \#8 with periodic root order $2^{6} \&$ twig length 2

\begin{tabular}{|c|r|}
\hline Order & Structure \\
\hline $2^{n+1}$ & $6 / 1$ \\
\hline
\end{tabular}

TABle 9 . Family \#9 with periodic root order $2^{5} \&$ twig length 1

\begin{tabular}{|c|r|}
\hline Order & Structure \\
\hline $2^{n+1}$ & $4 / 1$ \\
\hline
\end{tabular}

TABLE 10 . Family \#10 with periodic root order $2^{7} \&$ twig length 1 


\begin{tabular}{|l|r|r|r|r|r|r|r|r|r|r|r|r|}
\hline Order & \multicolumn{10}{|c|}{ Structure } \\
\hline $2^{n+1}$ & $24 / 1$ & & & & & & & & & & & \\
\hline $2^{n+2}$ & $8 / 4$ & & & & & & & & & & & \\
\hline $2^{n+3}$ & $16 / 8$ & $16 / 8$ & $4 / 2$ & $4 / 2$ & & & & & & & & \\
\hline $2^{n+4}$ & $24 / 12$ & $8 / 4$ & $8 / 4$ & $16 / 8$ & $12 / 6$ & $12 / 6$ & $12 / 6$ & $12 / 6$ & $16 / 8$ & $12 / 6$ & $8 / 4$ & $8 / 4$ \\
\hline & $12 / 6$ & $12 / 6$ & $24 / 12$ & $12 / 6$ & $32 / 16$ & $32 / 16$ & $32 / 16$ & $32 / 16$ & $\bullet$ & & & \\
\hline $2^{n+5}$ & 16 & 16 & 16 & 16 & $24 / 1$ & 16 & 16 & 24 & 24 & 16 & 16 & 32 \\
\hline & 32 & 32 & 32 & 32 & 32 & 32 & 32 & 16 & 16 & 16 & 16 & 32 \\
\hline & 16 & 32 & 16 & 32 & 32 & 24 & 24 & 24 & 24 & 32 & 32 & 24 \\
\hline & $24 / 1$ & 24 & 24 & 32 & 32 & 24 & 24 & 24 & 24 & 32 & 32 & 24 \\
\hline & 24 & 24 & 24 & 16 & 16 & 16 & 16 & 32 & 16 & $32 / 1$ & 16 & 32 \\
\hline & 32 & 24 & 24 & 24 & $24 / 1$ & 32 & 32 & 32 & 32 & 32 & 32 & 32 \\
\hline & 32 & 32 & 32 & 24 & 24 & 24 & 24 & 32 & 32 & 24 & 24 & 24 \\
\hline & 24 & 16 & 16 & 16 & 16 & 24 & 24 & 16 & 16 & 24 & 24 & 16 \\
\hline & 16 & 32 & 32 & 24 & 24 & 24 & 24 & 32 & 32 & 32 & 32 & 32 \\
\hline & 32 & 32 & 32 & 32 & 32 & 32 & 32 & 32 & 32 & 32 & 32 & 32 \\
\hline & 32 & 32 & 32 & 32 & 32 & 32 & 32 & 32 & 32 & 32 & 32 & 32 \\
\hline & 32 & 32 & 32 & 32 & 32 & 32 & 32 & 32 & 32 & 32 & 32 & 32 \\
\hline & 32 & 32 & 32 & 32 & 32 & 32 & 32 & 32 & 32 & 32 & 32 & 32 \\
\hline & 32 & 32 & 32 & 32 & 32 & 32 & 32 & 32 & 32 & 32 & 32 & \\
\hline
\end{tabular}

TABLE 11. Family \#11 with periodic root order $2^{14} \&$ twig length 5

\begin{tabular}{|c|c|c|c|c|c|c|c|c|c|c|c|c|c|c|c|c|c|c|c|c|}
\hline Order & \multicolumn{20}{|c|}{ Structure } \\
\hline $2^{n+1}$ & $12 / 4$ & & & & & & & & & & & & & & & & & & & \\
\hline $2^{n+2}$ & $18 / 6$ & $9 / 3$ & $18 / 6$ & $9 / 3$ & & & & & & & & & & & & & & & & \\
\hline $2^{n+3}$ & 12 & 12 & $18 / 6$ & 12 & 18 & 12 & 24 & 18 & 18 & 12 & 12 & \begin{tabular}{l|l}
2 & 18 \\
\end{tabular} & & 12 & 18 & 12 & 24 & 18 & \begin{tabular}{l|l}
3 & 18 \\
\end{tabular} & 8 \\
\hline $2^{n+4}$ & $6 / 2$ & 6 & 6 & 6 & 4 & 4 & $\bullet$ & & & & & & & & & & & & & \\
\hline $2^{n+5}$ & $6 / 2$ & & & & & & & & & & & & & & & & & & & \\
\hline $2^{n+6}$ & $12 / 4$ & $12 / 4$ & & & & & & & & & & & & & & & & & & \\
\hline $2^{n+7}$ & 24 & 24 & 24 & 24 & 24 & 24 & 24 & 24 & & & & & & & & & & & & \\
\hline
\end{tabular}

TABLE 12. Family \#12 with periodic root order $2^{11} \&$ twig length 3

\begin{tabular}{|c|c|c|c|c|c|c|c|c|c|c|c|c|c|c|c|c|c|c|}
\hline Order & \multicolumn{18}{|c|}{ Structure } \\
\hline $2^{n+1}$ & $20 / 8$ & & & & & & & & & & & & & & & & & \\
\hline $2^{n+2}$ & $10 / 4$ & $10 / 4$ & $10 / 4$ & $10 / 4$ & 2 & 4 & 4 & 4 & 4 & & & & & & & & & \\
\hline $2^{n+3}$ & $20 / 8$ & $12 / 4$ & 12 & 8 & $\varepsilon$ & 8 & & 8 & $\overline{12}$ & 12 & & & 12 & 12 & & 12 & & 12 \\
\hline $2^{n+4}$ & 4 & 4 & 4 & 4 & $10 / 2$ & \begin{tabular}{|l|l|}
4 & $10 /$
\end{tabular} & & $\mid 4$ & $10 / 4$ & $10 / 4$ & $10 /$ & & 6 & 6 & $\bullet$ & & & \\
\hline $2^{n+5}$ & $\overline{12}$ & 8 & $12 / 4$ & 6 & 6 & \begin{tabular}{|l|l}
6 & 1 \\
\end{tabular} & \begin{tabular}{l|l}
0 \\
\end{tabular} & 10 & 12 & 20 & & & 12 & 10 & 10 & & & 16 \\
\hline & 16 & 12 & 12 & 16 & 16 & 1 & & 12 & & & & & & & & & & \\
\hline $2^{n+6}$ & 6 & 6 & $6 / 2$ & $6 / 2$ & & & & & & & & & & & & & & \\
\hline $2^{n+7}$ & 24 & 24 & 24 & 24 & & & & & & & & & & & & & & \\
\hline
\end{tabular}

TABLE 13. Family \#13 with periodic root order $2^{12} \&$ twig length 4 


\begin{tabular}{|c|c|c|c|c|c|c|c|c|c|c|c|c|c|c|c|c|}
\hline Order & \multicolumn{16}{|c|}{ Structure } \\
\hline $2^{n+1}$ & $12 / 6$ & & & & & & & & & & & & & & & \\
\hline $2^{n+2}$ & 4 & 4 & $12 / 6$ & $8 / 4$ & $12 / 6$ & $8 / 4$ & $\bullet$ & & & & & & & & & \\
\hline $2^{n+3}$ & 3 & 3 & 6 & 6 & $8 / 4$ & $8 / 4$ & 6 & 6 & $6 / 3$ & $6 / 3$ & $12 / 6$ & $6 / 3$ & $6 / 3$ & 8 & 4 & $12 / 6$ \\
\hline & $12 / 6$ & 4 & 6 & & & & & & & & & & & & & \\
\hline $2^{n+4}$ & 4 & 4 & 8 & 4 & 4 & 4 & 8 & 4 & 8 & 6 & 6 & 8 & 6 & 6 & 4 & 4 \\
\hline & $12 / 6$ & $8 / 4$ & $12 / 6$ & $8 / 4$ & $8 / 4$ & 6 & 6 & $8 / 4$ & 6 & 6 & 4 & $\overline{4}$ & 4 & 4 & 4 & 4 \\
\hline & 4 & 4 & 8 & 8 & 8 & 8 & & & & & & & & & & \\
\hline $2^{n+5}$ & 4 & 4 & 8 & 8 & 4 & 8 & 8 & 8 & 6 & 4 & 3 & 3 & 6 & 6 & 4 & 8 \\
\hline & 6 & 6 & 6 & 6 & 16 & 16 & 16 & 16 & 16 & 16 & 16 & 16 & & & & \\
\hline
\end{tabular}

TABLE 14. Family \#14 with periodic root order $2^{10} \&$ twig length 4

\begin{tabular}{|c|r|r|r|r|r|r|r|r|r|r|r|r|r|r|r|r|}
\hline Order & \multicolumn{10}{|c|}{ Structure } \\
\hline $2^{n+1}$ & $4 / 2$ & & & & & & & & & & & & & & & \\
\hline $2^{n+2}$ & $8 / 4$ & $8 / 4$ & & & & & & & & & & & & & & \\
\hline $2^{n+3}$ & $16 / 8$ & $8 / 4$ & $8 / 4$ & $8 / 4$ & $8 / 4$ & $8 / 4$ & $16 / 8$ & $8 / 4$ & & & & & & & & \\
\hline $2^{n+4}$ & $16 / 2$ & $16 / 2$ & 8 & 8 & 16 & 16 & 8 & 8 & 16 & $16 / 2$ & 16 & 16 & 16 & 16 & 16 & 16 \\
\hline & 16 & 16 & 16 & 16 & 16 & 16 & 16 & 16 & 16 & 16 & 16 & 16 & 8 & 8 & 16 & 16 \\
\hline & 8 & 8 & 16 & 16 & 16 & 16 & 16 & 16 & $\bullet$ & & & & & & & \\
\hline $2^{n+5}$ & 4 & 4 & $4 / 2$ & 4 & 4 & & & & & & & & & & & \\
\hline $2^{n+6}$ & 8 & 8 & & & & & & & & & & & & & & \\
\hline
\end{tabular}

TABLE 15. Family \#15 with periodic root order $2^{11} \&$ twig length 4

\begin{tabular}{|c|r|r|r|r|r|r|r|r|r|l|l|l|l|l|l|l|l|}
\hline Order & \multicolumn{10}{|c|}{ Structure } \\
\hline $2^{n+1}$ & $4 / 2$ & & & & & & & & & & & & & & & & \\
\hline $2^{n+2}$ & $8 / 4$ & 8 & & & & & & & & & & & & & & & \\
\hline $2^{n+3}$ & $16 / 8$ & $16 / 8$ & 16 & 16 & & & & & & & & & & & & & \\
\hline $2^{n+4}$ & 16 & 16 & 16 & 16 & $16 / 1$ & $16 / 1$ & $16 / 1$ & $16 / 1$ & 16 & 16 & 16 & 16 & $16 / 1$ & $16 / 1$ & $16 / 1$ & $16 / 1$ & $\bullet$ \\
\hline $2^{n+5}$ & 4 & $4 / 2$ & 4 & 4 & 4 & 4 & 4 & & & & & & & & & & \\
\hline $2^{n+6}$ & 8 & 8 & & & & & & & & & & & & & & \\
\hline
\end{tabular}

TABLE 16. Family \#16 with periodic root order $2^{11} \&$ twig length 4

\begin{tabular}{|c|r|r|r|r|r|r|r|r|r|r|r|}
\hline Order & \multicolumn{10}{|c|}{ Structure } \\
\hline $2^{n+1}$ & $12 / 6$ & & & & & & & & & & \\
\hline $2^{n+2}$ & 6 & 6 & $6 / 3$ & 6 & 8 & 8 & & & & & \\
\hline $2^{n+3}$ & 4 & $4 / 2$ & $4 / 2$ & & & & & & & & \\
\hline $2^{n+4}$ & $12 / 6$ & 8 & 8 & $12 / 6$ & $\bullet$ & & & & & & \\
\hline $2^{n+5}$ & 16 & 16 & 12 & 8 & 8 & 8 & 8 & 8 & 8 & 16 & 16 \\
\hline
\end{tabular}

TABLE 17. Family \#17 with periodic root order $2^{10} \&$ twig length 4

\begin{tabular}{|c|r|r|r|}
\hline Order & \multicolumn{3}{|c|}{ Structure } \\
\hline $2^{n+1}$ & $12 / 1$ & & \\
\hline $2^{n+2}$ & $8 / 4$ & $\bullet$ & \\
\hline $2^{n+3}$ & 6 & 12 & 6 \\
\hline
\end{tabular}

TABLE 18. Family \#18 with periodic root order $2^{8} \&$ twig length 2 


\begin{tabular}{|c|r|r|r|r|r|r|r|r|r|r|}
\hline Order & \multicolumn{10}{|c|}{ Structure } \\
\hline $2^{n+1}$ & $4 / 2$ & & & & & & & & & \\
\hline $2^{n+2}$ & $4 / 2$ & $6 / 4$ & & & & & & & & \\
\hline $2^{n+3}$ & 2 & 2 & $4 / 2$ & $4 / 2$ & $4 / 2$ & $4 / 2$ & & & & \\
\hline $2^{n+4}$ & $6 / 4$ & $4 / 2$ & $6 / 2$ & $4 / 2$ & 2 & 1 & 1 & 2 & $\bullet$ & \\
\hline $2^{n+5}$ & $4 / 2$ & $4 / 2$ & $4 / 2$ & 2 & 2 & $4 / 2$ & $4 / 2$ & 2 & 2 & \\
\hline $2^{n+6}$ & $4 / 2$ & $2 / 1$ & 2 & 1 & 1 & 2 & 4 & 4 & $2 / 1$ & $2 / 1$ \\
\hline $2^{n+7}$ & $4 / 2$ & $4 / 2$ & 4 & 4 & 4 & & & & & \\
\hline $2^{n+8}$ & 6 & 4 & $2 / 1$ & $4 / 2$ & & & & & & \\
\hline $2^{n+9}$ & 4 & 4 & 4 & & & & & & & \\
\hline
\end{tabular}

TABLE 19. Family \#19 with periodic root order $2^{9} \&$ twig length 6

\begin{tabular}{|c|r|r|r|r|r|r|}
\hline Order & \multicolumn{7}{|c|}{ Structure } \\
\hline $2^{n+1}$ & $8 / 4$ & & & & & \\
\hline $2^{n+2}$ & $6 / 2$ & $6 / 2$ & 6 & 6 & & \\
\hline $2^{n+3}$ & $6 / 2$ & 3 & 4 & $3 / 1$ & & \\
\hline $2^{n+4}$ & $6 / 2$ & $6 / 2$ & 6 & $\bullet$ & & \\
\hline $2^{n+5}$ & $5 / 2$ & $4 / 2$ & $5 / 2$ & & & \\
\hline $2^{n+6}$ & 4 & 4 & 6 & 6 & 8 & 8 \\
\hline
\end{tabular}

TABLE 20. Family \#20 with periodic root order $2^{12} \&$ twig length 4

\begin{tabular}{|c|r|r|r|r|r|}
\hline Order & \multicolumn{5}{|c|}{ Structure } \\
\hline $2^{n+1}$ & $6 / 4$ & & & & \\
\hline $2^{n+2}$ & $6 / 4$ & 4 & $3 / 1$ & 2 & $\bullet$ \\
\hline $2^{n+3}$ & 4 & $6 / 2$ & 4 & 4 & \\
\hline $2^{n+4}$ & 4 & 2 & & & \\
\hline
\end{tabular}

TABLE 21. Family \#21 with periodic root order $2^{7} \&$ twig length 3

\begin{tabular}{|c|r|}
\hline Order & Structure \\
\hline $2^{n+1}$ & $4 / 1$ \\
\hline
\end{tabular}

TABLE 22. Family \#22 with periodic root order $2^{7} \&$ twig length 1

\begin{tabular}{|c|r|r|r|}
\hline Order & \multicolumn{3}{|c|}{ Structure } \\
\hline $2^{n+1}$ & $5 / 2$ & & \\
\hline $2^{n+2}$ & $5 / 2$ & $5 / 2$ & $\bullet$ \\
\hline $2^{n+3}$ & $4 / 1$ & $5 / 1$ & $4 / 1$ \\
\hline $2^{n+4}$ & $5 / 1$ & 4 & 2 \\
\hline $2^{n+5}$ & 4 & & \\
\hline
\end{tabular}

TABLe 23. Family \#23 with periodic root order $2^{7} \&$ twig length 4 


\begin{tabular}{|c|r|r|r|}
\hline Order & \multicolumn{3}{|c|}{ Structure } \\
\hline $2^{n+1}$ & $4 / 2$ & & \\
\hline $2^{n+2}$ & $6 / 2$ & 3 & $\bullet$ \\
\hline $2^{n+3}$ & $4 / 2$ & & \\
\hline $2^{n+4}$ & 3 & 4 & \\
\hline
\end{tabular}

TABle 24. Family \#24 with periodic root order $2^{7} \&$ twig length 3

\begin{tabular}{|c|r|}
\hline Order & Structure \\
\hline $2^{n+1}$ & $4 / 1$ \\
\hline
\end{tabular}

TABle 25 . Family \#25 with periodic root order $2^{7} \&$ twig length 1

\begin{tabular}{|l|r|r|}
\hline Order & \multicolumn{2}{|c|}{ Structure } \\
\hline $2^{n+1}$ & $6 / 2$ & $\bullet$ \\
\hline $2^{n+2}$ & 3 & \\
\hline
\end{tabular}

TABle 26 . Family \#26 with periodic root order $2^{7} \&$ twig length 2

\begin{tabular}{|c|r|}
\hline Order & Structure \\
\hline $2^{n+1}$ & $2 / 1$ \\
\hline
\end{tabular}

TABLE 27 . Family \#27 with periodic root order $2^{6} \&$ twig length 1

\begin{tabular}{|c|r|r|r|r|r|r|r|}
\hline Order & \multicolumn{7}{|c|}{ Structure } \\
\hline $2^{n+1}$ & $4 / 1$ & & & & & & \\
\hline $2^{n+2}$ & $8 / 2$ & & & & & & \\
\hline $2^{n+3}$ & $16 / 4$ & 16 & & & & & \\
\hline $2^{n+4}$ & $16 / 4$ & $16 / 4$ & 16 & 16 & $\bullet$ & & \\
\hline $2^{n+5}$ & $4 / 1$ & 4 & 4 & $4 / 1$ & $4 / 1$ & 4 & 4 \\
\hline $2^{n+6}$ & $8 / 2$ & 8 & 8 & & & & \\
\hline $2^{n+7}$ & 8 & 8 & & & & & \\
\hline
\end{tabular}

TABLE 28. Family \#28 with periodic root order $2^{9} \&$ twig length 4

\begin{tabular}{|c|r|r|r|r|r|r|r|r|}
\hline Order & \multicolumn{9}{|c|}{ Structure } \\
\hline $2^{n+1}$ & $16 / 2$ & & & & & & & \\
\hline $2^{n+2}$ & $12 / 4$ & $12 / 4$ & & & & & & \\
\hline $2^{n+3}$ & 12 & 24 & 24 & 12 & $24 / 1$ & 12 & 12 & 24 \\
\hline $2^{n+4}$ & $12 / 4$ & $\bullet$ & & & & & & \\
\hline $2^{n+5}$ & 8 & 8 & $16 / 2$ & & & & & \\
\hline $2^{n+6}$ & $12 / 4$ & $12 / 4$ & & & & & & \\
\hline $2^{n+7}$ & 12 & 24 & 24 & 12 & $24 / 1$ & 12 & 12 & 24 \\
\hline $2^{n+8}$ & 12 & & & & & & & \\
\hline
\end{tabular}

TABLE 29. Family \#29 with periodic root order $2^{12} \&$ twig length 5 


\begin{tabular}{|c|r|r|r|r|l|l|l|l|l|}
\hline Order & \multicolumn{9}{|c|}{ Structure } \\
\hline $2^{n+1}$ & $12 / 2$ & & & & & & & & \\
\hline $2^{n+2}$ & $16 / 2$ & $16 / 2$ & & & & & & & \\
\hline $2^{n+3}$ & $12 / 2$ & $12 / 2$ & $12 / 2$ & $12 / 2$ & & & & & \\
\hline $2^{n+4}$ & $24 / 4$ & 24 & 24 & 24 & 24 & 24 & 24 & 24 & $\bullet$ \\
\hline $2^{n+5}$ & $6 / 1$ & 12 & 6 & & & & & & \\
\hline $2^{n+6}$ & $16 / 2$ & & & & & & & & \\
\hline $2^{n+7}$ & 12 & 12 & & & & & & & \\
\hline
\end{tabular}

TABLE 30. Family \#30 with periodic root order $2^{11} \&$ twig length 4

\begin{tabular}{|c|c|c|c|c|c|c|c|c|c|}
\hline Order & \multicolumn{9}{|c|}{ Structure } \\
\hline $2^{n+1}$ & $16 / 4$ & & & & & & & & \\
\hline $2^{n+2}$ & $32 / 2$ & $32 / 2$ & 8 & 8 & & & & & \\
\hline $2^{n+3}$ & 16 & 16 & $16 / 4$ & $16 / 4$ & & & & & \\
\hline $2^{n+4}$ & 8 & 8 & $32 / 2$ & $32 / 2$ & 8 & 8 & $16 / 1$ & $16 / 1$ & - \\
\hline $2^{n+5}$ & $16 / 4$ & 8 & 8 & 16 & $16 / 4$ & & & & \\
\hline $2^{n+6}$ & $32 / 2$ & $32 / 2$ & 8 & 8 & 16 & 16 & 32 & 32 & \\
\hline $2^{n+7}$ & $16 / 4$ & $16 / 4$ & 16 & 16 & & & & & \\
\hline $2^{n+8}$ & 32 & 32 & 8 & 8 & 16 & 16 & 8 & 8 & \\
\hline
\end{tabular}

TABLE 31 . Family \#31 with periodic root order $2^{11} \&$ twig length 5

\begin{tabular}{|r|r|r|r|}
\hline Order & \multicolumn{3}{|c|}{ Structure } \\
\hline $2^{n+1}$ & $24 / 2$ & & \\
\hline $2^{n+2}$ & $12 / 2$ & $12 / 2$ & $\bullet$ \\
\hline $2^{n+3}$ & $24 / 1$ & 24 & 24 \\
\hline $2^{n+4}$ & $8 / 1$ & & \\
\hline $2^{n+5}$ & 16 & & \\
\hline
\end{tabular}

TABLE 32 . Family \#32 with periodic root order $2^{10} \&$ twig length 4

\begin{tabular}{|c|r|r|l|l|l|l|l|}
\hline Order & \multicolumn{7}{|c|}{ Structure } \\
\hline $2^{n+1}$ & $16 / 2$ & & & & & & \\
\hline $2^{n+2}$ & $8 / 4$ & $8 / 4$ & $\bullet$ & & & & \\
\hline $2^{n+3}$ & $8 / 1$ & 16 & 8 & $16 / 2$ & 8 & $8 / 1$ & 16 \\
\hline $2^{n+4}$ & 4 & 4 & 4 & $4 / 2$ & & & \\
\hline $2^{n+5}$ & 8 & 8 & & & & & \\
\hline
\end{tabular}

TABLe 33. Family \#33 with periodic root order $2^{10} \&$ twig length 4 


\begin{tabular}{|r|r|r|r|r|r|r|r|r|r|r|r|r|}
\hline Order & \multicolumn{10}{|c|}{ Structure } \\
\hline $2^{n+1}$ & $8 / 1$ & & & & & & & & & & & \\
\hline $2^{n+2}$ & $8 / 8$ & & & & & & & & & & & \\
\hline $2^{n+3}$ & 8 & 16 & 8 & 4 & 8 & 4 & 8 & $16 / 2$ & & & & \\
\hline $2^{n+4}$ & $8 / 8$ & 4 & $\bullet$ & & & & & & & & & \\
\hline $2^{n+5}$ & 4 & 4 & $8 / 1$ & $8 / 1$ & $8 / 1$ & 4 & 4 & & & & & \\
\hline $2^{n+6}$ & $8 / 8$ & $8 / 8$ & $8 / 8$ & & & & & & & & & \\
\hline $2^{n+7}$ & 8 & 16 & 8 & 4 & 8 & 4 & 8 & 16 & 4 & 8 & 16 & 8 \\
\hline & $16 / 2$ & 8 & 4 & 8 & 8 & 4 & 8 & 16 & 8 & 16 & 8 & 4 \\
\hline $2^{n+8}$ & 4 & 4 & & & & & & & & & & \\
\hline
\end{tabular}

TABLE 34. Family \#34 with periodic root order $2^{12} \&$ twig length 5

\begin{tabular}{|l|r|r|r|r|r|r|r|r|r|r|r|r|r|r|r|r|r|}
\hline Order & \multicolumn{10}{|c|}{ Structure } \\
\hline $2^{n+1}$ & $8 / 4$ & & & & & & & & & & & & & & & & \\
\hline $2^{n+2}$ & $8 / 1$ & $8 / 1$ & $8 / 1$ & $8 / 1$ & & & & & & & & & & & & & \\
\hline $2^{n+3}$ & $8 / 4$ & $8 / 4$ & $8 / 4$ & $8 / 4$ & & & & & & & & & & & & & \\
\hline $2^{n+4}$ & 8 & 16 & 8 & $16 / 8$ & 8 & 16 & 8 & 16 & 16 & 8 & 16 & 8 & 16 & 8 & 16 & 8 & $\bullet$ \\
\hline $2^{n+5}$ & 4 & 4 & 4 & 2 & $4 / 2$ & 4 & 2 & & & & & & & & & & \\
\hline $2^{n+6}$ & $8 / 1$ & $8 / 1$ & & & & & & & & & & & & & & & \\
\hline $2^{n+7}$ & 8 & 8 & & & & & & & & & & & & & & & \\
\hline
\end{tabular}

TABLE 35. Family \#35 with periodic root order $2^{11} \&$ twig length 4

\begin{tabular}{|c|r|r|r|r|}
\hline Order & \multicolumn{5}{|c|}{ Structure } \\
\hline $2^{n+1}$ & $12 / 3$ & & & \\
\hline $2^{n+2}$ & $12 / 3$ & 8 & 8 & $\bullet$ \\
\hline $2^{n+3}$ & 16 & 12 & & \\
\hline
\end{tabular}

TABLE 36 . Family \#36 with periodic root order $2^{8} \&$ twig length 2

\begin{tabular}{|c|r|r|}
\hline Order & \multicolumn{2}{|c|}{ Structure } \\
\hline $2^{n+1}$ & $16 / 1$ & \\
\hline $2^{n+2}$ & $8 / 2$ & $\bullet$ \\
\hline $2^{n+3}$ & 16 & \\
\hline
\end{tabular}

TABLE 37 . Family \#37 with periodic root order $2^{8} \&$ twig length 2

\begin{tabular}{|c|r|r|r|}
\hline Order & \multicolumn{3}{|c|}{ Structure } \\
\hline $2^{n+1}$ & $4 / 2$ & & \\
\hline $2^{n+2}$ & $8 / 2$ & 4 & $\bullet$ \\
\hline $2^{n+3}$ & $4 / 2$ & & \\
\hline $2^{n+4}$ & 4 & 8 & \\
\hline
\end{tabular}

TABLE 38 . Family \#38 with periodic root order $2^{7} \&$ twig length 3 


\begin{tabular}{|c|r|}
\hline Order & Structure \\
\hline $2^{n+1}$ & $8 / 1$ \\
\hline
\end{tabular}

TABle 39 . Family \#39 with periodic root order $2^{7} \&$ twig length 1

\begin{tabular}{|c|r|r|r|r|r|r|r|r|r|}
\hline Order & \multicolumn{10}{|c|}{ Structure } \\
\hline $2^{n+1}$ & $8 / 4$ & & & & & & & & \\
\hline $2^{n+2}$ & $4 / 4$ & $4 / 4$ & 4 & 4 & & & & & \\
\hline $2^{n+3}$ & $4 / 2$ & 4 & $4 / 2$ & 4 & 4 & $4 / 2$ & 4 & $4 / 2$ & \\
\hline $2^{n+4}$ & $4 / 2$ & 4 & 4 & 4 & 4 & 4 & $4 / 2$ & 4 & $\bullet$ \\
\hline $2^{n+5}$ & 8 & 8 & 8 & & & & & & \\
\hline
\end{tabular}

TABLE 40. Family \#40 with periodic root order $2^{12} \&$ twig length 5

\begin{tabular}{|c|r|r|r|r|r|r|r|r|r|r|r|r|r|}
\hline Order & \multicolumn{10}{|c|}{ Structure } \\
\hline $2^{n+1}$ & $12 / 4$ & & & & & & & & & & & & \\
\hline $2^{n+2}$ & 8 & $8 / 2$ & $8 / 2$ & 8 & & & & & & & & & \\
\hline $2^{n+3}$ & $12 / 4$ & $12 / 4$ & $8 / 2$ & $8 / 2$ & & & & & & & & & \\
\hline $2^{n+4}$ & $8 / 2$ & 8 & 8 & $8 / 2$ & 4 & 4 & 4 & 4 & 8 & 8 & $8 / 2$ & 8 & $\bullet$ \\
\hline $2^{n+5}$ & $12 / 4$ & $12 / 4$ & $12 / 4$ & 16 & 16 & & & & & & & & \\
\hline $2^{n+6}$ & 8 & 8 & 8 & 8 & 8 & $8 / 2$ & $8 / 2$ & 8 & 8 & 8 & 8 & 8 & \\
\hline $2^{n+7}$ & 12 & 12 & 8 & 8 & & & & & & & & & \\
\hline
\end{tabular}

TABLE 41 . Family \#41 with periodic root order $2^{12} \&$ twig length 5

\begin{tabular}{|c|r|r|r|r|l|}
\hline Order & \multicolumn{5}{|c|}{ Structure } \\
\hline $2^{n+1}$ & $9 / 4$ & & & & \\
\hline $2^{n+2}$ & $6 / 2$ & 6 & 6 & $6 / 2$ & $\bullet$ \\
\hline $2^{n+3}$ & $9 / 2$ & 9 & 9 & & \\
\hline $2^{n+4}$ & 4 & 4 & & & \\
\hline
\end{tabular}

TABLE 42 . Family \#42 with periodic root order $2^{10} \&$ twig length 3

\begin{tabular}{|c|r|r|r|r|r|r|r|r|}
\hline Order & \multicolumn{7}{|c|}{ Structure } \\
\hline $2^{n+1}$ & $6 / 4$ & & & & & & & \\
\hline $2^{n+2}$ & 4 & $4 / 4$ & $4 / 4$ & 4 & $\bullet$ & & & \\
\hline $2^{n+3}$ & $3 / 2$ & 6 & 3 & $6 / 4$ & 3 & $3 / 2$ & 6 & \\
\hline $2^{n+4}$ & 2 & 2 & 2 & 2 & 2 & 2 & 2 & 2 \\
\hline
\end{tabular}

TABLE 43 . Family \#43 with periodic root order $2^{12} \&$ twig length 4

\begin{tabular}{|c|r|r|l|}
\hline Order & \multicolumn{3}{|c|}{ Structure } \\
\hline $2^{n+1}$ & $6 / 2$ & & \\
\hline $2^{n+2}$ & 4 & $4 / 2$ & $\bullet$ \\
\hline $2^{n+3}$ & 6 & & \\
\hline
\end{tabular}

TABLE 44 . Family \#44 with periodic root order $2^{8} \&$ twig length 2 


\begin{tabular}{|c|r|r|r|}
\hline Order & \multicolumn{3}{|c|}{ Structure } \\
\hline $2^{n+1}$ & $6 / 2$ & & \\
\hline $2^{n+2}$ & $6 / 2$ & $4 / 1$ & $\bullet$ \\
\hline $2^{n+3}$ & $6 / 2$ & $6 / 1$ & \\
\hline $2^{n+4}$ & $4 / 1$ & $6 / 1$ & 8 \\
\hline $2^{n+5}$ & 6 & 8 & \\
\hline
\end{tabular}

TABle 45 . Family \#45 with periodic root order $2^{8} \&$ twig length 4

\begin{tabular}{|c|r|r|r|}
\hline Order & \multicolumn{3}{|c|}{ Structure } \\
\hline $2^{n+1}$ & $2 / 2$ & & \\
\hline $2^{n+2}$ & $4 / 4$ & 2 & $\bullet$ \\
\hline $2^{n+3}$ & 2 & 2 & $2 / 2$ \\
\hline $2^{n+4}$ & 2 & 2 & \\
\hline
\end{tabular}

TABLe 46 . Family \#46 with periodic root order $2^{7} \&$ twig length 3

\begin{tabular}{|c|r|}
\hline Order & Structure \\
\hline $2^{n+1}$ & $6 / 1$ \\
\hline
\end{tabular}

TABLE 47 . Family \#47 with periodic root order $2^{7} \&$ twig length 1

\begin{tabular}{|c|r|r|}
\hline Order & \multicolumn{2}{|c|}{ Structure } \\
\hline $2^{n+1}$ & $3 / 2$ & $\bullet$ \\
\hline $2^{n+2}$ & 2 & \\
\hline
\end{tabular}

TABle 48 . Family \#48 with periodic root order $2^{6} \&$ twig length 2

\begin{tabular}{|c|r|}
\hline Order & Structure \\
\hline $2^{n+1}$ & $2 / 1$ \\
\hline
\end{tabular}

TABLE 49 . Family \#49 with periodic root order $2^{5} \&$ twig length 1

\begin{tabular}{|c|r|r|}
\hline Order & \multicolumn{2}{|c|}{ Structure } \\
\hline $2^{n+1}$ & $3 / 2$ & $\bullet$ \\
\hline $2^{n+2}$ & 1 & \\
\hline
\end{tabular}

TABLE 50. Family \#50 with periodic root order $2^{5} \&$ twig length 2

\begin{tabular}{|c|r|}
\hline Order & Structure \\
\hline $2^{n+1}$ & $2 / 1$ \\
\hline
\end{tabular}

TABLE 51 . Family \#51 with periodic root order $2^{7} \&$ twig length 1

\begin{tabular}{|c|r|}
\hline Order & Structure \\
\hline $2^{n+1}$ & $2 / 1$ \\
\hline
\end{tabular}

TABLE 52. Family \#52 with periodic root order $2^{7} \&$ twig length 1 


\begin{tabular}{|c|r|r|}
\hline Order & \multicolumn{2}{|c|}{ Structure } \\
\hline $2^{n+1}$ & $3 / 2$ & $\bullet$ \\
\hline $2^{n+2}$ & $2 / 1$ & \\
\hline $2^{n+3}$ & 1 & \\
\hline
\end{tabular}

TABLE 53. Family \#53 with periodic root order $2^{7} \&$ twig length 3

\begin{tabular}{|c|r|}
\hline Order & Structure \\
\hline $2^{n+1}$ & $6 / 1$ \\
\hline
\end{tabular}

TABLE 54. Family \#54 with periodic root order $2^{6} \&$ twig length 1

\begin{tabular}{|c|r|}
\hline Order & Structure \\
\hline $2^{n+1}$ & $10 / 1$ \\
\hline
\end{tabular}

TABLE 55. Family \#55 with periodic root order $2^{6} \&$ twig length 1

\begin{tabular}{|c|r|l|l|l|}
\hline Order & \multicolumn{4}{|c|}{ Structure } \\
\hline $2^{n+1}$ & $16 / 4$ & & & \\
\hline $2^{n+2}$ & 4 & $8 / 1$ & 4 & 8 \\
\hline
\end{tabular}

TABLE 56. Family \#56 with periodic root order $2^{7} \&$ twig length 2

\begin{tabular}{|c|r|r|r|}
\hline Order & \multicolumn{3}{|c|}{ Structure } \\
\hline $2^{n+1}$ & $12 / 2$ & & \\
\hline $2^{n+2}$ & $12 / 2$ & $6 / 1$ & $\bullet$ \\
\hline $2^{n+3}$ & $12 / 2$ & 4 & \\
\hline $2^{n+4}$ & 4 & 4 & \\
\hline
\end{tabular}

TABLE 57. Family \#57 with periodic root order $2^{7} \&$ twig length 3

\begin{tabular}{|c|r|}
\hline Order & Structure \\
\hline $2^{n+1}$ & $3 / 1$ \\
\hline
\end{tabular}

TABLE 58. Family \#58 with periodic root order $2^{4} \&$ twig length 1

\begin{tabular}{|c|r|}
\hline Order & Structure \\
\hline $2^{n+1}$ & $6 / 1$ \\
\hline
\end{tabular}

TABLE 59. Family \#59 with periodic root order $2^{4} \&$ twig length 1

\begin{tabular}{|c|r|r|}
\hline Order & \multicolumn{2}{|c|}{ Structure } \\
\hline $2^{n+1}$ & $4 / 2$ & $\bullet$ \\
\hline $2^{n+2}$ & 2 & \\
\hline
\end{tabular}

TABLE 60 . Family \#60 with periodic root order $2^{6} \&$ twig length 2 


\begin{tabular}{|c|r|}
\hline Order & Structure \\
\hline $2^{n+1}$ & $7 / 1$ \\
\hline
\end{tabular}

TABLE 61 . Family \#61 with periodic root order $2^{6} \&$ twig length 1

\begin{tabular}{|c|c|c|c|c|}
\hline Order & \multicolumn{4}{|c|}{ Structure } \\
\hline $2^{n+1}$ & $16 / 4$ & & & \\
\hline $2^{n+2}$ & $24 / 1$ & 24 & 12 & 12 \\
\hline
\end{tabular}

TABLE 62 . Family \#62 with periodic root order $2^{7} \&$ twig length 2

\begin{tabular}{|c|r|r|r|r|}
\hline Order & \multicolumn{5}{|c|}{ Structure } \\
\hline $2^{n+1}$ & $18 / 3$ & & & \\
\hline $2^{n+2}$ & $18 / 3$ & 14 & 16 & $\bullet$ \\
\hline $2^{n+3}$ & 12 & 8 & & \\
\hline
\end{tabular}

TABLE 63 . Family \#63 with periodic root order $2^{7} \&$ twig length 2

\begin{tabular}{|c|r|}
\hline Order & Structure \\
\hline $2^{n+1}$ & $13 / 1$ \\
\hline
\end{tabular}

TABLE 64 . Family \#64 with periodic root order $2^{6} \&$ twig length 1

\begin{tabular}{|c|r|}
\hline Order & Structure \\
\hline $2^{n+1}$ & $5 / 1$ \\
\hline
\end{tabular}

TABLE 65 . Family \#65 with periodic root order $2^{6} \&$ twig length 1

\begin{tabular}{|c|r|r|}
\hline Order & \multicolumn{2}{|c|}{ Structure } \\
\hline $2^{n+1}$ & $6 / 2$ & $\bullet$ \\
\hline $2^{n+2}$ & 2 & \\
\hline
\end{tabular}

TABLE 66 . Family \#66 with periodic root order $2^{6} \&$ twig length 2

\begin{tabular}{|c|r|r|}
\hline Order & \multicolumn{2}{|c|}{ Structure } \\
\hline $2^{n+1}$ & $3 / 2$ & $\bullet$ \\
\hline $2^{n+2}$ & 2 & \\
\hline
\end{tabular}

TABLE 67 . Family \#67 with periodic root order $2^{5} \&$ twig length 2

\begin{tabular}{|c|r|}
\hline Order & Structure \\
\hline $2^{n+1}$ & $5 / 1$ \\
\hline
\end{tabular}

TABLE 68 . Family \#68 with periodic root order $2^{6} \&$ twig length 1

\begin{tabular}{|l|r|r|}
\hline Order & \multicolumn{2}{|c|}{ Structure } \\
\hline $2^{n+1}$ & $8 / 2$ & $\bullet$ \\
\hline $2^{n+2}$ & 2 & \\
\hline
\end{tabular}

TABLE 69 . Family \#69 with periodic root order $2^{6} \&$ twig length 2 


\begin{tabular}{|c|r|}
\hline Order & Structure \\
\hline $2^{n+1}$ & $2 / 1$ \\
\hline
\end{tabular}

TABle 70 . Family \#70 with periodic root order $2^{5} \&$ twig length 1

\begin{tabular}{|c|r|}
\hline Order & Structure \\
\hline $2^{n+1}$ & $12 / 1$ \\
\hline
\end{tabular}

TABLE 71 . Family \#71 with periodic root order $2^{6} \&$ twig length 1

\begin{tabular}{|c|l|l|}
\hline Order & \multicolumn{2}{|l|}{ Structure } \\
\hline $2^{n+1}$ & $12 / 2$ & \\
\hline $2^{n+2}$ & $20 / 1$ & 16 \\
\hline
\end{tabular}

TABLE 72 . Family \#72 with periodic root order $2^{7} \&$ twig length 2

\begin{tabular}{|c|r|}
\hline Order & Structure \\
\hline $2^{n+1}$ & $9 / 1$ \\
\hline
\end{tabular}

TABLE 73 . Family \#73 with periodic root order $2^{6} \&$ twig length 1

\begin{tabular}{|c|r|}
\hline Order & Structure \\
\hline $2^{n+1}$ & $9 / 1$ \\
\hline
\end{tabular}

TABLE 74 . Family \#74 with periodic root order $2^{6} \&$ twig length 1

\begin{tabular}{|l|r|r|}
\hline Order & \multicolumn{2}{|c|}{ Structure } \\
\hline $2^{n+1}$ & $6 / 2$ & $\bullet$ \\
\hline $2^{n+2}$ & 2 & \\
\hline
\end{tabular}

TABLE 75 . Family \# 75 with periodic root order $2^{5} \&$ twig length 2

\begin{tabular}{|c|r|r|}
\hline Order & \multicolumn{2}{|c|}{ Structure } \\
\hline $2^{n+1}$ & $6 / 2$ & $\bullet$ \\
\hline $2^{n+2}$ & 2 & \\
\hline
\end{tabular}

TABLE 76 . Family \#76 with periodic root order $2^{5} \&$ twig length 2

\begin{tabular}{|c|r|}
\hline Order & Structure \\
\hline $2^{n+1}$ & $6 / 1$ \\
\hline
\end{tabular}

TABLE 77 . Family \#77 with periodic root order $2^{5} \&$ twig length 1

\begin{tabular}{|c|r|}
\hline Order & Structure \\
\hline $2^{n+1}$ & $6 / 1$ \\
\hline
\end{tabular}

TABLE 78 . Family \#78 with periodic root order $2^{6} \&$ twig length 1 


\begin{tabular}{|c|r|}
\hline Order & Structure \\
\hline $2^{n+1}$ & $4 / 1$ \\
\hline
\end{tabular}

TABLE 79 . Family \#79 with periodic root order $2^{5} \&$ twig length 1

\begin{tabular}{|c|r|}
\hline Order & Structure \\
\hline $2^{n+1}$ & $4 / 1$ \\
\hline
\end{tabular}

TABLE 80 . Family \#80 with periodic root order $2^{5} \&$ twig length 1

\begin{tabular}{|c|r|}
\hline Order & Structure \\
\hline $2^{n+1}$ & $10 / 1$ \\
\hline
\end{tabular}

TABLE 81 . Family \#81 with periodic root order $2^{5} \&$ twig length 1

\section{REFERENCES}

N. Blackburn (1958), "On a special class of p-groups", Acta Math., 100, 45-92. MR 21:1349 Wieb Bosma and John Cannon (1997), Handbook of MAgma functions. School of Mathematics and Statistics, Sydney University.

Harold Brown, Rolf Bülow, Joachim Neubüser, Hans Wondratschek and Hans Zassenhaus (1978), Crystallographic Groups of Four-Dimensional Space. Wiley-Interscience, New York. MR 58:4109 J.H. Conway, R.T. Curtis, S.P. Norton, R.A. Parker and R.A. Wilson (1985), Atlas of finite groups. Clarendon Press, Oxford. MR 88g:20025

Helga Finken (1979), "Raumgruppen und Serien von $p$-Gruppen". Staatsexamensarbeit, RWTH Aachen.

B. Huppert (1967), Endliche Gruppen I, Grundlehren Math. Wiss., 134. Springer-Verlag, Berlin, New York. MR 37:302

Bertram Huppert and Norman Blackburn (1982), Finite Groups II, Grundlehren Math. Wiss., 242. Springer-Verlag, Berlin, New York. MR 84i:20001a

Rodney James (1975), "2-groups of almost maximal class", J. Austral. Math. Soc. Ser. A, 19, 343-357. MR 52:3318

Rodney James (1983), "2-groups of almost maximal class: corrigendum", J. Austral. Math. Soc. Ser. A, 35, 307. MR 84i:20019

C.R. Leedham-Green and M.F. Newman (1980), "Space groups and groups of prime-power order I", Arch. Math. (Basel), 35, 193-202. MR 81m:20029

C.R. Leedham-Green and S. McKay (1984), "On the classification of $p$-groups of maximal class", Quart. J. Math. Oxford Ser. (2), 35, 293-304. MR 85m:20027

C.R. Leedham-Green, S. McKay and W. Plesken (1986a), "Space groups and groups of primepower order. V. A bound to the dimension of space groups with fixed coclass", Proc. London Math. Soc. (3), 52, 73-94. MR 87g:20036

C.R. Leedham-Green, S. McKay and W. Plesken (1986b), "Space groups and groups of prime power order. VI. A bound to the dimension of a 2-adic space group with fixed coclass", J. London Math. Soc. (2), 34, 417-425. MR 87m:20017

C.R. Leedham-Green (1994), "The structure of finite p-groups", J. London Math. Soc. (2), 50, 49-67. MR 95j:20022a

Roger C. Lyndon (1985), Groups and Geometry, London Math. Soc. Lecture Note Ser., 101. Cambridge University Press, London. MR 87i:20068

S. McKay (1994), "The precise bound to the coclass of space groups", J. London Math. Soc. (2), 50, 488-500. MR 95m:20017

M.F. Newman (1977), "Determination of groups of prime-power order", Group Theory, Lecture Notes in Math., 573, (Canberra, 1975), pp. 73-84. Springer-Verlag, Berlin, Heidelberg, New York. MR 56:12115

M.F. Newman (1990), "Groups of prime-power order", Groups-Canberra 1989, Lecture Notes in Math., 1456, pp. 49-62. Springer-Verlag, Berlin, Heidelberg, New York. MR 91m:20031 
M.F. Newman and E.A. O'Brien (1996), "Application of computers to questions like those of Burnside, II", Internat. J. Algebra Comput. 6, 593-605. MR 97k:20002

E.A. O'Brien (1990), "The p-group generation algorithm", J. Symbolic Comput., 9, 677-698. MR 91j:20050

E.A. O'Brien (1994), "Isomorphism testing for p-groups", J. Symbolic Comput., 17, 133-147. MR 95f:20040b

Martin Schönert et al. (1997), GAP - Groups, Algorithms and Programming. Lehrstuhl D für Mathematik, RWTH Aachen.

Axel Schneider (1997), "Classifying $p$-groups by Coclass", M.Math. thesis, University of New South Wales.

Aner Shalev (1994), "The structure of finite $p$-groups: effective proof of the coclass conjectures", Invent. Math., 115, 315-345. MR 95j:20022b

A. Shalev (1995), "Finite $p$-groups", Finite and locally finite groups, NATO Adv. Sci. Inst. Ser. C Math. Phys. Sci., 471, (Istanbul, 1994), pp. 401-450. Kluwer Acad. Publ., Dordrecht.

A. Shalev and E.I. Zel'manov (1992), "Pro-p groups of finite coclass", Math. Proc. Cambridge Philos. Soc., 111, 417-421. MR 93e:20030

Charles C. Sims (1994), Computation with finitely presented groups. Cambridge University Press. MR 95f:20053

School of Mathematical Sciences, Australian National University, Canberra, ACT 0200, Australia

E-mail address: newman@maths.anu.edu.au

Department of Mathematics, University of Auckland, Private Bag 92019, Auckland, New ZeALAND

E-mail address: obrien@math.auckland.ac.nz 